September 2004

CERN-PH-TH/2004-183

\title{
Aspects of Split Supersymmetry
}

\author{
N. Arkani-Hamed ${ }^{1}$, S. Dimopoulos ${ }^{2}$, G.F. Giudice ${ }^{3}$, A. Romanino ${ }^{3}$ \\ 1 Jefferson Laboratory of Physics, Harvard University, \\ Cambridge, Massachusetts 02138, USA \\ 2 Physics Department, Stanford University, \\ Stanford, California 94305, USA \\ 3 CERN, Theory Division, \\ CH-1211 Geneva 23, Switzerland
}

\begin{abstract}
We explore some fundamental differences in the phenomenology, cosmology and model building of Split Supersymmetry compared with traditional low-scale supersymmetry. We show how the mass spectrum of Split Supersymmetry naturally emerges from theories where the dominant source of supersymmetry breaking preserves an $R$ symmetry, characterize the class of theories where the unavoidable $R$-breaking by gravity can be neglected, and point out a new possibility, where supersymmetry breaking is directly communicated at tree level to the visible sector via renormalizable interactions. Next, we discuss possible low-energy signals for Split Supersymmetry. The absence of new light scalars removes all the phenomenological difficulties of low-energy supersymmetry, associated with one-loop flavor and CP violating effects. However, the electric dipole moments of leptons and quarks do arise at two loops, and are automatically at the level of present limits with no need for small phases, making them accessible to several ongoing new-generation experiments. We also study proton decay in the context of Split Supersymmetry, and point out scenarios where the dimension-six induced decays may be observable. Finally, we show that the novel spectrum of Split Supersymmetry opens up new possibilities for the generation of dark matter, as the decays of ultraheavy gravitinos in the early universe typically increase the abundance of the lightest neutralino above its usual freeze-out value. This allows for lighter gauginos and Higgsinos, more accessible both to the LHC and to dark-matter detection experiments.
\end{abstract}




\section{Introduction}

Our concept of Naturalness - the principle that Nature abhors fine-tunings - is based on theories with a few vacua. It has led to the proposal of low-scale supersymmetry, in order to avoid tuning short-distance parameters to 30-decimals. But this principle faces a serious challenge from the cosmological constant problem, where we see no new physics at the scale $\sim 10^{-3} \mathrm{eV}$ required by naturalness.

Recent developments in string theory [1] suggest the existence of an enormous "landscape" of long-lived metastable vacua. These can have a significant impact on the criterion of naturalness, and may provide the key to resolving the cosmological constant problem along the lines of ref. [2]. They may also have a bearing on the solution to the hierarchy problem. For example, if the number of vacua that break supersymmetry at high-scale is more than $10^{30}$ times larger than those with low-scale supersymmetry, then the breaking of supersymmetry at high scales is favored [3]. The 30-decimal tuning is compensated by the enormous "entropy factor" favoring high-scale breaking of supersymmetry. In this case, the simplest possibility would be that the Standard Model (SM) is preferred to the usual low-energy supersymmetric SM [4]. This would then account for why we have not seen any evidence for supersymmetry - either in the spectrum or in rare process, such as FCNCs, CP violation, proton decay, etc. - at the expense of giving up two major successes of the supersymmetric SM: gauge coupling unification [5] and natural dark-matter candidate [6]. A more interesting possibility that preserves these successes is that approximate chiral symmetries protect the fermions of the supersymmetric SM down to the TeV scale [7, 8]. So, the sparticle spectrum of these theories is "split" in two: (i) the scalars (squarks and sleptons) that get a mass at the high-scale of supersymmetry breaking $\tilde{m}$, which can be as large as the GUT scale, and (ii) the fermions (gauginos and higgsinos) which remain near the electroweak scale and can account for both gauge-coupling unification and DM. The only light scalar in this theory is a finely-tuned Higgs. So, rather than the dull prediction that the LHC will discover just the Higgs, these theories - coined Split Supersymmetry - predict gauginos and higgsinos at a $\mathrm{TeV}$, maintain the successes of the supersymmetric SM, and account for the absence of problematic flavor and CP-violation, of fast proton decay, and of an excessively light Higgs, caused by the presence of light squarks and sleptons in the supersymmetric SM.

In this paper we address some novel theoretical, cosmological and phenomenological aspects of Split Supersymmetry ${ }^{1}$. An important theoretical issue is how the spectrum of Split Supersymmetry can naturally emerge from a high-energy theory. In sects. 2 and 3 we

\footnotetext{
${ }^{1}$ Some consequences of Split Supersymmetry have been considered in ref. [9].
} 
characterize the general class of theories that have this property. We also present in sect. 4 models where the supersymmetry breaking is directly mediated at tree level to the visible sector of the theory.

Having abandoned naturalness, the crucial ingredient pinning the gaugino and Higgsino masses to the $\mathrm{TeV}$ scale is the requirement for the lightest supersymmetric particle (LSP) to form the dark matter of the universe. Novel properties of the gravitino in Split Supersymmetry can impact this analysis. In sect. 5 we discuss the properties of gravitinos in Split Supersymmetry, emphasizing their impact on the issue of dark matter. Gravitino decay generates a non-thermal population of dark-matter neutralinos, which gives an additional contribution to the energy density on top of the usual freeze-out abundance. This, in general, suggests that the mass of the supersymmetric particles can be lighter than what derived from the thermal abundances - making Split Supersymmetry potentially more accessible to the LHC and to dark-matter detection experiments.

While the decisive verdict on Split Supersymmetry awaits TeV scale collider experiments, it is important to examine what signals can show up in other, low-energy experiments. Having eliminated the new scalars, the rate for anomalous effects in FCNC processes, which are troublesome for usual low-energy supersymmetry, are predicted to be unobservably small. Similarly, the proton decay rates from dimension 5 operators become irrelevant - these problems are eliminated so efficiently that there are no associated signals to be seen. However, in sect. 6] we show that in Split Supersymmetry, the contribution to lepton and quark electric dipole moments (EDM), arising at two loops, are naturally of the order of the current experimental limits for $\mathrm{CP}$ violating phases of order unity. This is to be contrasted with usual low-energy supersymmetry, where CP violation is a problematic feature, with electric dipole moments arising at one-loop being too large by roughly a factor of $10^{2}-10^{3}$ for softterm phases of order one. The naturally observable EDM are an exciting generic prediction of Split Supersymmetry, and may be checked by a new generation of experiments that plan to improve the limit by a factor of at least $\sim 100-1000$, possibly before the turn-on of the LHC. We also study proton decay in Split Supersymmetry in sect. [7, and point out scenarios under which the dimension-6 mediated decays may be observable.

In sect. 8 we conclude with a detailed summary of our results and remarks on directions for future research. 


\section{Split Supersymmetry and the Pattern of Supersym- metry Breaking}

The spectrum of Split Supersymmetry is defined by squark masses and $B_{\mu}$ term generated at a large mass scale $\tilde{m}$, and by gaugino masses and $\mu$ term of the order of the weak scale. In this section we want to characterize the conditions under which the mechanism of supersymmetry breaking leads to such a spectrum, rather than the usual case in which all supersymmetric partners are approximately mass-degenerate. The question is reminiscent of the $\mu$ problem: why isn't $\mu=\mathcal{O}\left(M_{\mathrm{Pl}}\right)$ generated in the exactly supersymmetric theory? In Split Supersymmetry we are encountering an extended $\mu$ problem: why aren't gaugino masses and $\mu=\mathcal{O}(\tilde{m})$ generated in the broken supersymmetric theory?

At first sight, it may appear that the ordinary case of mass-degenerate superpartners is the most generic since, after supersymmetry is broken, all these particles are expected to acquire masses. We want to show that this is not necessarily the case. The nature of the mass spectrum depends on the presence or the absence of an approximate (or accidental) $R$-symmetry of the observable sector of the theory. Once this symmetry is realized, the mass spectrum of Split Supersymmetry naturally emerges.

It is known [10] that there is a connection between the existence of $R$-symmetries in the hidden sector and of supersymmetry breaking. If the relevant superpotential is a generic function of fields (i.e. all interaction terms are present with no tuned coefficients), the presence of an $R$-symmetry is a necessary condition for supersymmetry breaking, while a spontaneously broken $R$-symmetry provides a sufficient condition. However, because of the nonrenormalization theorem in supersymmetry, it is not unusual to encounter superpotentials that are non-generic functions of fields, where certain interaction terms are absent. Many example of such superpotentials, leading to spontaneously-broken supersymmetry, are known. Moreover, $R$-symmetry cannot be an exact symmetry once the theory is extended to supergravity and the cancellation of the cosmological constant is allowed. Indeed, such cancellation is obtained by tuning the gravitino mass to a value proportional to the scalar component of the superpotential $W$. Since $R[W]=2$, the condition of vanishing cosmological constant necessarily leads to a breaking of the $R$-symmetry.

Let us turn to consider the various patterns of supersymmetry breaking, paying special attention to the properties of the underlying $R$-symmetry. 


\section{$2.1 \quad$ F-breaking}

We start by reviewing standard results regarding the origin of the soft terms, in order to introduce notations and to allow a comparison with the analysis presented in the next section.

Supersymmetry breaking is parametrized by a spurion chiral superfield

$$
X=1+\theta^{2} \tilde{m} .
$$

The superfield $X$ breaks supersymmetry and $R$-symmetry, since both its scalar and auxiliary components have a background value. Masses for the scalar components of visible-sector superfields $Q\left(\tilde{m}_{Q}\right)$, gaugino masses $\left(M_{\tilde{g}}\right)$, and trilinear $A$-terms are generated by the operators

$$
\begin{aligned}
\int d^{4} \theta X^{\dagger} X Q^{\dagger} Q & \rightarrow \tilde{m}_{Q}^{2}=\tilde{m}^{2}, \\
\int d^{2} \theta X W_{\alpha} W_{\alpha} & \rightarrow M_{\tilde{g}}=\tilde{m}, \\
\int d^{2} \theta X Q^{3} & \rightarrow A=\tilde{m} .
\end{aligned}
$$

Here $W_{\alpha}$ is the gauge superfield strength, and $Q^{3}$ is a gauge-invariant combination of visiblesector superfields. All soft terms are of the order of the mass scale $\tilde{m}$. Notice that, in the absence of supersymmetry breaking $(\tilde{m} \rightarrow 0)$, the interactions in eqs. (2) -(4) are $R$-invariant, once we assign $R[X]=0$. Therefore, supersymmetry and $R$-symmetry are simultaneously broken by the auxiliary component $F_{X}=\tilde{m}$.

For phenomenological reasons, we have to exclude the operator

$$
\int d^{2} \theta M_{*} H_{1} H_{2},
$$

where $H_{1,2}$ are the two Higgs-doublet superfields and $M_{*}$ is a mass scale characteristic of the exact-supersymmetry theory. This can be achieved by: (i) a global $U(1) P Q$ symmetry with $P Q\left[H_{1} H_{2}\right] \neq 0$; or (ii) an $R$-symmetry with $R\left[H_{1} H_{2}\right] \neq 2$; or (iii) a mechanism that does not allow to embed this term in the underlying GUT. Once this is done, supersymmetry breaking generates the $\mu$-term and $B_{\mu}$ (the scalar counterpart of the $\mu$-term) through the operators 11 .

$$
\begin{aligned}
\int d^{4} \theta X^{\dagger} H_{1} H_{2} & \rightarrow \mu=\tilde{m}, \\
\int d^{4} \theta X^{\dagger} X H_{1} H_{2} & \rightarrow B_{\mu}=\tilde{m}^{2}, \mu=\tilde{m} .
\end{aligned}
$$

These interactions are allowed, in the supersymmetric limit, by a combination of the $R$ and $P Q$ symmetries such that the $R$ charges are $R\left[H_{1} H_{2}\right]=0$. Consistently, this symmetry forbids the unwanted operator in eq. (5). In conclusion, all possible soft terms (including $\mu$ ) have been generated at the scale $\tilde{m}$. 


\subsection{D-breaking}

Next, we want to examine the case in which supersymmetry breaking does not lead to $R$-symmetry breaking. Therefore we consider the spurion superfield

$$
Y=1+\theta^{4} \tilde{m}^{2}
$$

which breaks supersymmetry, but preserves an $R$-symmetry if we assign $R[Y]=0$. This spurion could arise from a genuine supersymmetry $D$-breaking in the gauge sector. However, it could also be induced by a chiral superfield $X$, with $\langle X\rangle=0$ and $\left\langle F_{X}\right\rangle \neq 0$. As an example, this happens in the simplest of all theories of supersymmetry breaking, with a linear superpotential for a chiral superfield $X$

$$
W=\mu^{2} X
$$

This has $F_{X}=\mu^{2}$ and broken supersymmetry. The vev of $X$ is undetermined at this level; however, if there are higher dimension operators in the Kähler potential of the form

$$
\delta K=-\frac{\left(X^{\dagger} X\right)^{2}}{M^{2}}
$$

then $X$ is stabilized at the origin $\langle X\rangle=0$, with $m_{X}^{2}=\mu^{4} / M^{2}>0$. (In fact, precisely this sort of term in the Kähler potential is generated at 1-loop in renormalizable O'Raifeartaigh models of supersymmetry breaking). If such a field $X$ appears in the interactions with visiblesector fields only in the combination $X^{\dagger} X$, then the visible sector will have supersymmetry breaking without $R$-breaking. In particular, this happens if $X$ is a non-singlet under a hidden-sector gauge group (as is often the case in models with dynamical supersymmetry breaking) or if there exists a discrete symmetry $X \rightarrow-X$. For simplicity, we will call "D-breaking" the case characterized by eq. (8). However, it should be clear that we do not necessarily require that supersymmetry breaking is triggered by a gauge superfield, but rather that the supersymmetry breaking is not accompanied by $R$ breaking.

Couplings of the spurion $Y$ to the visible sector induce the soft terms

$$
\begin{aligned}
\int d^{4} \theta Y Q^{\dagger} Q & \rightarrow \tilde{m}_{Q}^{2}=\tilde{m}^{2} \\
\int d^{4} \theta Y H_{1} H_{2} & \rightarrow B_{\mu}=\tilde{m}^{2}
\end{aligned}
$$

No other renormalizable operator is allowed. Notice that we have defined our spurions as dimensionless, and so we are effectively working to all orders in $Y$. Only visible-sector fields determine the operator dimensionality. 
Since we have excluded the operator in eq. (5), possibly by a GUT mechanism, the visible sector has an accidental $R$-symmetry with $R[Y]=0$ and $R\left[H_{1} H_{2}\right]=0$, even after supersymmetry is broken. This is the symmetry that forbids the appearance of gaugino masses, $A$ and $\mu$ terms. This symmetry is accidental, since it has not been imposed on the theory, but it is just the consequence of supersymmetry, gauge symmetry, field dimensionality, and the absence of the operator in eq. (5), once D-breaking is assumed. Notice, in particular, that imposing on the theory an exact $R$-symmetry is not justified since, as we have previously discussed, the cancellation of the cosmological constant necessarily implies an explicit $R$ breaking.

The situation is analogous to how lepton number is implemented in the SM. Lepton number is only an accidental SM symmetry and it is expected to be violated in the fundamental (possibly GUT) theory by effects of order unity. The smallness of the neutrino mass is then explained not by small coefficients, but by the large scale hierarchy between the electroweak and GUT masses. Similarly, in $D$-breaking, even if the $R$-symmetry is broken in the fundamental theory by effects of order unity, gaugino masses and the $\mu$ parameter will be suppressed with respect to squark masses and $B_{\mu}$, whenever there is a certain hierarchy between the fundamental (possibly Planck) scale and $\tilde{m}$.

Equations (11) and (12) allow for the possibility of a cancellation of the Higgs mass term. In particular, a Kähler structure of the kind

$$
\int d^{4} \theta Y\left(\sin \beta H_{1}-\cos \beta H_{2}^{\dagger}\right)\left(\sin \beta H_{1}^{\dagger}-\cos \beta H_{2}\right)
$$

leads to a massless Higgs superfield, for any value of $\beta$. Although the cancellation needs a fine-tuning, it is interesting that Split Supersymmetry allows this possibility for the Higgs mass and not, for instance, for squark masses, so there can be a reason why of all the scalars of the supersymmetric SM, only the Higgs is fine-tuned to be light.

To generate the other soft terms, we have to include the leading non-renormalizable operators,

$$
\begin{aligned}
\frac{1}{M_{*}} \int d^{4} \theta Y W_{\alpha} W_{\alpha} r & \rightarrow M_{\tilde{g}}=\frac{\tilde{m}^{2}}{M_{*}} r, \\
\frac{1}{M_{*}} \int d^{4} \theta Y Q^{3} r & \rightarrow A=\frac{\tilde{m}^{2}}{M_{*}} r, \\
\frac{1}{M_{*}} \int d^{4} \theta Y D^{2}\left(H_{1} H_{2}\right) r & \rightarrow \mu=\frac{\tilde{m}^{2}}{M_{*}} r .
\end{aligned}
$$

Here $M_{*}$ is the mass that characterizes the interactions between hidden and visible sectors. In supergravity, we identify $M_{*}$ with $M_{\mathrm{Pl}}$, but smaller values of $M_{*}$ are possible in alternative 
schemes of supersymmetry-breaking mediation. The mass spectrum of Split Supersymmetry naturally emerges from $D$-breaking, whenever $\tilde{m} \ll M_{*}$. The parameter $r$ that we have included in eqs. (14)-(16) measures a possible further suppression caused by an (approximate) $R$-symmetry present in the dynamics that mediates supersymmetry breaking at the scale $M_{*}$. It could be viewed as a scalar spurion field carrying an $R$ charge. Indeed, if $R[r]=-2$, the interactions in eqs. (14)-(16) are $R$ invariant. If the underlying dynamics does not preserve an $R$-symmetry, we can set $r=\mathcal{O}(1)$.

Next, we can establish what is the reasonable minimum value of $r$ in theories where there is an energy range for which the 4-D supergravity description is valid. This is given by the effect of gravitational interactions that communicate to the visible sector the unavoidable $R$ violation coming from the cancellation of the cosmological constant. In supergravity, the positive vacuum energy associated with supersymmetry breaking is cancelled by having a non-zero expectation value for the superpotential $W$ which contributes $-3|W|^{2}$ to the vacuum energy. As $W$ has $R$-charge 2 , this breaks $R$, and gives a gravitino mass

$$
m_{3 / 2}=e^{\frac{K}{2 M_{\mathrm{Pl}}^{2}}} \frac{|W|}{M_{\mathrm{Pl}}^{2}} .
$$

Here $M_{\mathrm{Pl}}=2.4 \times 10^{18} \mathrm{GeV}$ is the reduced Planck mass. We can get a reasonable lower bound on $m_{3 / 2}$ as follows: given scalar masses of order $\tilde{m}$, there are contributions to the vacuum energy of at least order $\sim \tilde{m}^{4} /\left(16 \pi^{2}\right)$, and therefore we expect at least

$$
\frac{|W|^{2}}{M_{\mathrm{Pl}}^{2}} \gtrsim \frac{\tilde{m}^{4}}{16 \pi^{2}} \Rightarrow m_{3 / 2} \gtrsim \frac{\tilde{m}^{2}}{4 \pi M_{\mathrm{Pl}}} .
$$

Now, in order to generate gaugino masses, we must break both $R$ and supersymmetry. The minimum amount of $R$-breaking will come from the non-zero $W$. Local operators of the form

$$
\int d^{4} \theta \frac{W_{\alpha} W_{\alpha} W^{\dagger}\left(Q^{\dagger} Q+\cdots\right)}{M_{*}^{6}},
$$

where $Q$ are generic chiral fields in the visible sector, can not be protected by any symmetries, and indeed we expect gravitational loops to generate such operators as counterterms with $M_{*} \sim M_{\mathrm{Pl}}$. Now, only given the supersymmetry breaking of the heavy scalar masses $\tilde{m}$, we can expect that the terms $Q^{\dagger} Q+\cdots$ have a non-vanishing $\theta^{2} \bar{\theta}^{2}$ component of size comparable to the vacuum energy of the low-energy theory beneath $\tilde{m}$, which is $\sim \frac{\tilde{m}^{4}}{16 \pi^{2}}$, leading to

$$
M_{\tilde{g}} \gtrsim \frac{\tilde{m}^{6}}{M_{\mathrm{Pl}}^{5}} \sim \frac{m_{3 / 2}^{3}}{M_{\mathrm{Pl}}^{2}},
$$

ignoring loop factors. Barring cancellations, this can be taken as a plausible minimum value of the gaugino mass, since it is the result of just the existence of gravity and the cancellation 
of the cosmological constant. This magnitude can be estimated diagrammatically in terms of a one-loop gravitational contribution to gaugino masses (induced by graviton/gaugino and gravitino/gauge-boson exchange), which is cutoff in the ultraviolet by the supersymmetrybreaking mass $m_{3 / 2}$. The phenomenological requirement that eq. (20) does not exceed the $\mathrm{TeV}$ gives the constraint $m_{3 / 2} \lesssim 10^{13} \mathrm{GeV}$. Incidentally, we remark that in the case of gravity mediation $\left(\tilde{m} \sim m_{3 / 2}\right)$ this bound is numerically equivalent to the one derived by the condition that the gluino lifetime is shorter than the age of the universe, which is necessary to evade the experimental limits from searches of anomalously heavy isotopes [7]. Finally, notice that considerations similar to those we have made for gaugino masses also apply to the $\mu$ term. A Higgs-gravitino loop with a $B_{\mu}$ mass insertion induces $\mu \sim m_{3 / 2}^{3} / M_{\mathrm{Pl}}^{2}$, taking the gravity-mediation result $B_{\mu} \sim m_{3 / 2}^{2}$.

We have seen that there is some connection between the gaugino/Higgsino and gravitino masses, as they both break $R$. Is there any relation between $\tilde{m}$ and $m_{3 / 2}$ ? In fact these can usefully be thought of as the independent parameters characterizing a model of Split Supersymmetry. There are theories where $\tilde{m} \ll m_{3 / 2}$ - this occurred in one of the models in ref. [7, where the gravitino mass $m_{3 / 2}$ came from Scherk-Schwartz breaking with a fifth dimension as $m_{3 / 2} \sim 1 / R$, while the scalar masses were $\tilde{m} \sim \frac{1}{R^{2} M_{\mathrm{Pl}}}$. Using the fifth dimension to lower the higher-dimensional Planck scale to the GUT scale actually predicted $m_{3 / 2} \sim$ $1 / R \sim 10^{13} \mathrm{GeV}$, gaugino/Higgsinos right at the TeV scale, and $\tilde{m} \sim 10^{9} \mathrm{GeV}$. There are also theories in which $\tilde{m} \gg m_{3 / 2}$, as in the models of direct mediation we discuss in sect. 4 ,

\section{Contributions from Anomaly Mediation}

We have argued that the breaking of $R$ required by the cancellation of the vacuum energy in supergravity will eventually infect the visible sector, generating gaugino masses of order at least $M_{\tilde{g}} \sim m_{3 / 2}^{3} / M_{\mathrm{Pl}}^{2}$. However, there is a potentially larger effect, coming from anomaly mediation [12, which typically generates $M_{\tilde{g}} \sim \alpha /(4 \pi) m_{3 / 2}$. For $m_{3 / 2} \lesssim 100 \mathrm{TeV}$ or so, this will be subdominant to whatever generates the gaugino masses, but what happens for $m_{3 / 2} \gg M_{\tilde{g}}$ ? In ref. [7, a concrete model of supersymmetry breaking using a fifth dimension was constructed, where the anomaly-mediated contributions were small, with $m_{3 / 2} \sim 10^{13}$ $\mathrm{GeV}$, in fact predicting gaugino and Higgsino masses near $\sim 100 \mathrm{GeV}$. Our purpose here is to explore the issue in more general terms: why do we get the anomaly-mediated contribution, and under what circumstances is it naturally suppressed? 
Let us review the origin of the anomaly-mediated contribution to gaugino masses

$$
M_{\tilde{g}}=\frac{\beta(g)}{g} F_{\phi},
$$

where $\beta(g)=d g / d \ln \mu$ and $\phi=1+\theta^{2} F_{\phi}$ is the conformal compensator, which appears in the Lagrangian in form

$$
\mathcal{L}=\int d^{4} \theta \phi^{\dagger} \phi K+\left(\int d^{2} \theta \phi^{3} W+\text { h.c. }\right) .
$$

In order to see what the induced $F_{\phi}$ is, we set $K, W$ equal to their expectation values in the supersymmetry-breaking background (with $\left.K\right|_{0}=-3 M_{\mathrm{Pl}}^{2}$ to canonically normalize the Einstein action), and in components we find that the potential is

$$
V_{\mathrm{vac}}=3 M_{\mathrm{Pl}}^{2}\left|F_{\phi}\right|^{2}+\left(F_{\phi}^{*} A+\text { h.c. }\right)+V \text {. }
$$

Here

$$
A=-\left.3 W^{*}\right|_{0}-\left.K\right|_{\theta^{2}}, \quad V=-\left.K\right|_{\theta^{4}}-\left.2 \operatorname{Re} W\right|_{\theta^{2}},
$$

and the gravitino mass is

$$
\left|m_{3 / 2}\right|=\frac{|W|}{M_{\mathrm{Pl}}^{2}} .
$$

The equation of motion for $F_{\phi}$ now simply determines

$$
F_{\phi}=-\frac{A}{3 M_{\mathrm{Pl}}^{2}}=m_{3 / 2}+\frac{\left.K\right|_{\theta^{2}}}{3 M_{\mathrm{Pl}}^{2}},
$$

and this gives the vacuum energy

$$
V_{\mathrm{vac}}=V-\frac{|A|^{2}}{3 M_{\mathrm{Pl}}^{2}} .
$$

Fine-tuning the vacuum energy to zero, we also find that

$$
\left|F_{\phi}\right|=\sqrt{\frac{V}{3 M_{\mathrm{Pl}}^{2}}} .
$$

In theories in which supersymmetry is broken dynamically without singlets in the hidden sector, the term $\left.K\right|_{\theta^{2}} /\left(3 M_{\mathrm{Pl}}^{2}\right)$ in eq. (26) is negligible, and we obtain $\left|F_{\phi}\right|=m_{3 / 2}$. In general, however, $F_{\phi}$ is not proportional to $m_{3 / 2}$, but it is directly determined by $V$ as in eq. (28).

In a theory where supersymmetry is broken already in the global limit, $V$ can be thought of as the "vacuum energy" of the supersymmetry breaking sector. More precisely, it is the Goldstino decay constant; the Lagrangian for the Goldstino $\chi$ has a quadratic part

$$
V \bar{\chi} \bar{\sigma}^{\mu} \partial_{\mu} \chi+\cdots
$$


Thus in theories where supersymmetry breaking is decoupled from gravity, so that $V$ and $\left.K\right|_{\theta^{2}}$ remain constant as $M_{\mathrm{Pl}} \rightarrow \infty$, we have $\left|F_{\phi}\right|=m_{3 / 2}$. However, this is not the case for theories where supersymmetry breaking is intimately tied to gravity and supersymmetry is restored as $M_{\mathrm{Pl}} \rightarrow \infty$, so that $V \rightarrow 0$ in this limit. This may appear at first sight to be a sick limit, since the coefficient of the Goldstino kinetic term is going to zero. But in fact, in such models, the Goldstino does get a kinetic term via mixing with the gravitino. This is the secret of the no-scale supergravity structure, and also helps explain why the no-scale structure is so ubiquitous in compactifications of theories with extra dimensions. As is familiar, the scalar radius moduli $T$ only acquire kinetic terms by mixing with gravity, and the same is true for their fermionic partners $\psi_{T}$. Indeed, we can see this easily in a theory with a single extra dimension [13] with a radius modulus $T=r+\theta \psi_{T}+\cdots$. Since $M_{\mathrm{Pl}}^{2}=2 M_{5}^{3} \operatorname{Re}(r)$ we have

$$
K=-3 M_{5}^{3}\left(T+T^{\dagger}\right)
$$

and we can add a constant superpotential

$$
W=M_{5}^{3} c
$$

It is easy to see that $V=0$ and $F_{\phi}=0$, since both $\left.K\right|_{\theta^{4}}$ and $\left.W\right|_{\theta^{2}}$ vanish, while

$$
F_{T}=c^{*}, \quad m_{3 / 2}=\frac{c M_{5}^{3}}{M_{\mathrm{Pl}}^{2}}
$$

and supersymmetry is broken. This is equivalent [14 to a Scherk-Schwarz breaking with $c$ setting the phase picked up by the gravitino traversing the dimension.

At this level the modulus $r$ is undetermined; in any case the Kähler potential will be modified by quantum corrections, and we may also have additional terms in the superpotential. Suppose then that the Kähler and superpotentials are modified by introducing the functions $k\left(T+T^{\dagger}\right)$ and $w(T)$ as follows

$$
K=-3 M_{5}^{3}\left(T+T^{\dagger}\right)+k\left(T+T^{\dagger}\right), W=c M_{5}^{3}+w(T) .
$$

Let us work to first order in $k, w$. By computing the equation of motion for $F_{T}$, we find

$$
F_{\phi}=\frac{c^{*} k^{\prime \prime}+w^{\prime *}}{3 M_{5}^{3}}=\frac{|c|^{2} k^{\prime \prime}+w^{*} c}{3 m_{3 / 2} M_{\mathrm{Pl}}^{2}} .
$$

While the potential is

$$
V_{\mathrm{vac}}(T)=V_{K}+V_{W},
$$

where

$$
V_{K}=-|c|^{2} k^{\prime \prime}, V_{W}=-2 \operatorname{Re}\left(w^{\prime} c^{*}\right) .
$$


Note that

$$
-\operatorname{Re}\left(\frac{F_{\phi}}{m_{3 / 2}^{*}}\right)=\frac{V_{K}+\frac{1}{2} V_{W}}{3\left|m_{3 / 2}\right|^{2} M_{\mathrm{Pl}}^{2}}=\frac{V_{\mathrm{vac}}(T)+V_{K}}{6\left|m_{3 / 2}\right|^{2} M_{\mathrm{Pl}}^{2}} \rightarrow \frac{V_{K}}{6\left|m_{3 / 2}\right|^{2} M_{\mathrm{Pl}}^{2}},
$$

where in the last expression we have put in the fine-tuning to make $V_{\text {vac }}(T)$ vanish at the minimum of the potential. From here we can conclude that

$$
\left|\frac{F_{\phi}}{m_{3 / 2}}\right| \geq\left|\operatorname{Re}\left(\frac{F_{\phi}}{m_{3 / 2}^{*}}\right)\right|=\frac{V_{K}}{6\left|m_{3 / 2}\right|^{2} M_{\mathrm{Pl}}^{2}}
$$

The inequality above is saturated when there are no relative phases between $F_{\phi}$ and $m_{3 / 2}^{*}$. We typically expect that the real and imaginary parts of $F_{\phi}$ are comparable, so the right hand side of the above is a good estimate for the actual size of $\left|F_{\phi}\right|$.

What can we expect for the range of possible natural values for $V_{K}$ and thus $\left|F_{\phi}\right|$ ? Clearly, $V_{K}$ gets a contribution at least from the one-loop vacuum energy of the non-supersymmetric theory beneath the gravitino mass. Barring cancellations, this yields a reasonable lower bound of

$$
V_{K \min } \sim \frac{m_{3 / 2}^{4}}{16 \pi^{2}} .
$$

An upper bound on $V_{K}$ is of order $\sim m_{3 / 2}^{2} M_{\mathrm{Pl}}^{2}$, and therefore we can bound

$$
\frac{m_{3 / 2}^{3}}{16 \pi^{2} M_{\mathrm{Pl}}^{2}} \lesssim\left|F_{\phi}\right| \lesssim m_{3 / 2}
$$

The lower bound will be saturated in any theory where supersymmetry breaking shuts off above the mass of the gravitino. An example is provided by the model of ref. [7, where the gravitino mass is about $1 / r$ and the scale above which supersymmetry breaking shuts off is also $1 / r$, with the radius stabilized by balancing Casimir energies scaling as $1 / r^{4} \sim m_{3 / 2}^{4}$. Note that, when this lower bound is saturated, the anomaly-mediated contribution to gaugino masses is subdominant to the direct gravitational contribution we discussed in the previous section.

The expression for $F_{\phi}$ in eq. (38) has a nice physical interpretation. The Goldstino field here is the fermionic component of $T$; defining $\psi_{T}=\left\langle F_{T}\right\rangle \chi=c^{*} \chi$ (to first order), under a supersymmetry transformation $\delta_{\zeta} \chi=\zeta$. The quadratic part of the Goldstino kinetic term is then, to leading order,

$$
-|c|^{2} k^{\prime \prime} \bar{\chi} \bar{\sigma}^{\mu} \partial_{\mu} \chi=V_{K} \bar{\chi} \bar{\sigma}^{\mu} \partial_{\mu} \chi
$$

so we can identify $V_{K}$ with the Goldstino decay constant. The link between $F_{\phi}$ and the Goldstino decay constant is not an accident, and it is illuminating to understand its origin 
without any reference to the conformal compensator formalism, directly at component level. Suppose we have somehow broken supersymmetry and cancelled the vacuum energy to get flat space. The gravitino $\psi_{\mu}$ is massive and its quadratic action is of the form

$$
M_{\mathrm{Pl}}^{2}\left(\epsilon^{\mu \nu \alpha \beta} \bar{\psi}_{\mu} \bar{\sigma}_{\nu} \partial_{\alpha} \psi_{\beta}+m_{3 / 2} \psi_{\mu} \sigma^{\mu \nu} \psi_{\nu}\right)
$$

Of course, the massive gravitino has extra degrees of freedom - the longitudinal polarizations - compared to the massless gravitino. This is reflected in the fact that the above Lagrangian is no longer invariant under the gauge transformation $\delta_{\zeta} \psi_{\mu}=\partial_{\mu} \zeta$ that were used to reduce the degrees of freedom described by $\psi_{\mu}$ to the two transverse polarizations. As familiar in ordinary gauge theories, it is useful to explicitly introduce the longitudinal modes. This can simply be done by performing the broken-gauge transformation and promoting the transformation parameter to a field. For massive gauge bosons, for instance, we have the familiar

$$
f^{2} A_{\mu} A^{\mu} \rightarrow f^{2}\left(A_{\mu}+\partial_{\mu} \theta\right)^{2} \supset f^{2}\left(\partial_{\mu} \theta\right)^{2}
$$

and we see that the gauge boson mass term turns into the kinetic term of the "eaten" longitudinal mode $\theta$, which shifts under the gauge symmetry.

Doing the same thing for the gravitino, we have

$$
m_{3 / 2} \psi_{\mu} \sigma^{\mu \nu} \psi_{\nu} \rightarrow m_{3 / 2}\left(\partial_{\mu} \chi+\psi_{\mu}\right) \sigma^{\mu \nu}\left(\partial_{\nu} \chi+\psi_{\nu}\right)
$$

Already we notice a significant difference with the gauge theory case - the only possible kinetic term for the Goldstino $\chi$ appears to be of the form $(\partial \chi)^{2}$, which is sick for a fermion. However, due to the antisymmetry of $\sigma^{\mu \nu}$, this kinetic term actually vanishes (which is in fact what dictates the use of $\sigma^{\mu \nu}$ rather than $\eta^{\mu \nu}$ in the gravitino mass term). The Goldstino here does indeed get a healthy kinetic term, but only by mixing with gravity. We can now couple the gravitino to all the other fields of the theory, including all massive regulator fields, in the standard way, and at this level there is no induced supersymmetry breaking anywhere else in the spectrum. This analysis is however puzzling if we think of a theory that breaks supergravity already in the globally supersymmetric limit. There, the Goldstino exists independently of gravity! How do we describe this situation? After all, in the unitary gauge we still have a massive gravitino described by the same effective Lagrangian.

The resolution is that the gravitino transformation property under supersymmetry is changed to

$$
\delta \psi_{\mu}=\partial_{\mu} \zeta+i f \sigma_{\mu} \bar{\zeta}
$$

Before saying anything about the size of $f$, we can identify $f$ with $F_{\phi}$ in the conformal compensator formalism. Because of the modified transformation law for $\psi_{\mu}$, if we now 
couple the gravitino to other fields, what used to be supersymmetric will no longer be so, and the variation can only be compensated by adding supersymmetry breaking terms to the matter Lagrangian from the outset. It is easy to see that the new term in the variation of $\psi_{\mu}$ is simply the superpartner of a conformal transformation (compare $\left.\delta h_{\mu \nu}=\eta_{\mu \nu} \phi\right)$, and the required change of the Lagrangian is conveniently made by turning on $F_{\phi}=f$ in a conformal compensator field.

With the modified transformation, in promoting $\zeta$ to the Goldstino field $\chi$, we find the quadratic kinetic term for $\chi$

$$
V_{K} \bar{\chi} \bar{\sigma}^{\mu} \partial_{\mu} \chi+\cdots
$$

where the Goldstino decay constant $V_{K}$ is

$$
V_{K}=|f|^{2} M_{\mathrm{Pl}}^{2}+2 \operatorname{Re}\left(m_{3 / 2} M_{\mathrm{Pl}}^{2} f\right)=M_{\mathrm{Pl}}^{2}\left(\left|f+m_{3 / 2}^{*}\right|^{2}-\left|m_{3 / 2}\right|^{2}\right)
$$

Inverting this, we can bound $\left|f / m_{3 / 2}\right|$ in terms of $V_{K}$ as

$$
\left|\frac{f}{m_{3 / 2}}\right| \geq \sqrt{1+\frac{V_{K}}{m_{3 / 2}^{2} M_{\mathrm{Pl}}^{2}}}-1 .
$$

The above expression then bounds $F_{\phi}=f$ directly in terms of physical quantities: the Goldstino decay constant $V_{K}$ and the gravitino mass $m_{3 / 2}$. Again in theories when all the relevant parameters are real, the inequality above becomes an equality.

Note as before that in theories in which supersymmetry is already broken in the limit of global supersymmetry, $V_{K}=3 m_{3 / 2}^{2} M_{\mathrm{Pl}}^{2}$ and eq. (48) becomes

$$
\frac{\left|F_{\phi}\right|}{m_{3 / 2}} \rightarrow 1 .
$$

On the other hand, in theories where the Goldstino dominantly gets its kinetic term by mixing with the gravitino, $V_{K} \ll m_{3 / 2}^{2} M_{\mathrm{Pl}}^{2}$, and eq. (48) gives

$$
\frac{\left|F_{\phi}\right|}{m_{3 / 2}} \rightarrow \frac{V_{g}}{2 m_{3 / 2}^{2} M_{\mathrm{Pl}}^{2}} \ll 1 .
$$

Summarizing, then, we have a simple characterization of the size of anomaly mediation: in any theory where supersymmetry is broken by non-gravitational dynamics, so that the supersymmetry breaking survives taking $M_{\mathrm{Pl}} \rightarrow \infty$, anomaly mediation will persist, but in theories where supersymmetry breaking is tied to gravity and the Goldstino gets it kinetic term by mixing with gravity, $F_{\phi}$ and anomaly mediation are suppressed. 
Even when $F_{\phi}$ is comparable to $m_{3 / 2}$, the supersymmetric SM fields can be insulated from anomaly-mediated soft masses. An example [15] is provided by the theories where the SM fields descend from a broken CFT or, in an AdS/CFT dual language, live on an IR brane of a warped compactification. There is now a new dynamical field - the dilaton $\mathrm{S}$ of the broken CFT - and the Kähler and superpotentials for the SM superfields have the structure

$$
K=e^{S+S^{\dagger}} \hat{K}, \quad W=e^{3 S} \hat{W} .
$$

Including the conformal compensator, the Lagrangian has the form

$$
\int d^{4} \theta\left(-3 M_{\mathrm{Pl}}^{2} \phi^{\dagger} \phi+\phi^{\dagger} \phi e^{S+S^{\dagger}} \hat{K}\right)+\left(\int d^{2} \theta \phi^{3} e^{3 S} \hat{W}+\text { h.c. }\right) \text {. }
$$

We can now define a new field $\omega=\phi e^{S}$, which then decouples $\phi$ from direct communication from the SM sector

$$
\int d^{4} \theta\left(-3 M_{\mathrm{Pl}}^{2} \phi^{\dagger} \phi+\omega^{\dagger} \omega \hat{K}\right)+\left(\int d^{2} \theta \omega^{3} \hat{W}+\text { h.c. }\right) .
$$

Now, even breaking supersymmetry in some generic way generating $F_{\phi}$, there is no anomalymediated contribution to the soft terms. Of course again the dilaton $\omega$ must be stabilized, but the form of the stabilization and the resulting size of the supersymmetry breaking proportional to $F_{\phi}$ are model-dependent. It is clear that we can use this mechanism to arbitrarily shield the visible sector from the breaking of supergravity, which is why ref. [15] dubbed this observation "supersymmetry without supergravity". In particular, we can again consistently making $m_{3 / 2} \gg M_{\tilde{g}}$.

\section{Direct Mediation of Supersymmetry Breaking}

One of the ubiquitous features of usual theories of supersymmetry breaking is the presence of a hidden sector where supersymmetry is broken, and only indirectly mediated to the visible fields. It is obviously simpler to imagine that the supersymmetric SM fields pick up a mass directly at tree-level, by renormalizable interactions with a supersymmetry breaking sector. However, this hope was dashed very early on. First, with only $F$-term breaking, the only supersymmetry breaking masses for chiral superfields are $B_{\mu}$ type terms, which makes it impossible to make all the scalars heavy - in particular there is always a scalar lighter than the up quark [16]. The addition of new gauge factors opens up the possibility of using $D$-terms. However, there is a bigger problem: tree-level breaking can generate scalar masses, but not gaugino masses, which will then necessarily be suppressed at least by a loop factor 
relative to the scalars. This forces the scalars so heavy that a tuning of at least $\sim 10^{-3}$ is re-introduced for electroweak symmetry breaking.

The situation is clearly different in Split Supersymmetry, where we are allowed to consider gauginos much lighter than the heavy scalars. Thus, the possibility of direct mediation can be re-examined in this context. The goal is to find a theory where squarks and sleptons pick up a mass directly at tree level from the supersymmetry breaking sector. If the supersymmetry breaking preserves an $R$-symmetry, the gauginos and Higgsinos can only acquire masses from higher dimension operators suppressed by a scale $M_{*}$. As long as $M_{*}$ is sufficiently smaller than $M_{\mathrm{Pl}}$, all the physics associated with supergravity and in particular the gravitational breaking of the $R$-symmetry are irrelevant; in such models, the gravitino mass can naturally be comparable or even smaller than gaugino and Higgsino masses.

We can illustrate this general idea with a simple model. Since we want to generate $m^{2} Q^{\dagger} Q$ type masses for the supersymmetric SM scalars at tree-level, the SM fields must be charged under a new gauge symmetry that gets a $D$-term. So, let us begin with the simplest example of a supersymmetry breaking theory of this form. Consider a $U(1)$ gauge symmetry and chiral superfields $X, Z, \phi, \phi^{c}, Y, Y^{c}$ with $U(1)$ charges $0,0,-1,1,-1,1$ respectively, together with other possible fields $\psi_{i}$ with charges $q_{i}$. The superpotential contains

$$
W=\lambda X\left(\phi \phi^{c}-m^{\prime 2}\right)+m \phi^{c} Y+\lambda^{\prime} \phi Y^{c} Z
$$

and $m, m^{\prime}$ are some comparable mass scales. This theory spontaneously breaks supersymmetry: the $Y$ equation of motion forces $\phi^{c}=0$, which is in conflict with the $X$ equation and D-flatness for the $U(1)$. It is easy to analyze the physics in a limit where we imagine $m^{\prime} \gg m$. The $U(1)$ gauge symmetry is broken and we have a vector multiplet of mass $\mathrm{gm}^{\prime}$, and $Y^{c}, Z$ also pair up to get a mass $\lambda^{\prime} m^{\prime}$, while the superpotential for $Y$ is

$$
W=m^{\prime} m Y,
$$

clearly breaking supersymmetry. Integrating out the massive vector multiplet at tree-level also generates new terms in the Kähler potential for $Y$ and the $\psi_{i}$. It is easiest to see this in superspace: ignoring the $1 / g^{2} W_{\alpha}^{2}$ kinetic term for the gauge field, and going to unitary gauge, we have in the Kähler potential

$$
K=m^{\prime 2}\left(V^{2}+\cdots\right)+Y^{\dagger}(1+V+\cdots) Y+\psi_{i}^{\dagger}\left(1+q_{i} V+\cdots\right) \psi_{i}
$$

and we can trivially integrate out $V$ to obtain the correction

$$
-\frac{Y^{\dagger} Y}{2 m^{\prime 2}}\left(\frac{Y^{\dagger} Y}{2}+q_{i} \psi_{i}^{\dagger} \psi_{i}\right) \text {. }
$$


The superpotential forces

$$
F_{Y}=m m^{\prime}
$$

and this in turn generates soft masses for the $Y$ and $\psi_{i}$ from the corrected Kähler potential

$$
m_{Y}^{2}=\frac{m^{2}}{2}, \quad m_{\psi_{i}}^{2}=\frac{q_{i} m^{2}}{2} .
$$

Of course these are nothing but the $D$-term contributions to the soft masses of these charged fields, although it is more appropriate to simply integrate out the heavy field, rather than using a description with the vev of a heavy multiplet. Note importantly that $m_{Y}^{2}>0$, so that $Y$ is stabilized at $Y=0$, preserving an $R$-symmetry with the obvious charges. Also, note that if we had any couplings of $X$ to other fields, say of the form

$$
W=h X H_{u} H_{d}
$$

then there are induced couplings to $Y$ in the low-energy effective theory, which can be seen simply by noting that this coupling can be shifted into a redefinition of $m$ as $m^{2} \rightarrow$ $m^{2}+h / \lambda H_{u} H_{d}$, and so we have

$$
W=m m^{\prime} Y \rightarrow \sqrt{m^{\prime 2}+H_{u} H_{d}} m Y=m m^{\prime} Y+\frac{m}{2 m^{\prime}} Y H_{u} H_{d} .
$$

The fields $H_{u}, H_{d}$ then pick up a $B_{\mu}$ soft term of the order of

$$
B_{\mu}=\frac{m^{2}}{2} \sim m_{\psi_{i}}^{2} .
$$

This can be simply thought of as arising from a non-vanishing $F_{X}$.

We now wish to directly couple this sector to the supersymmetric SM. Clearly, we cannot only have the SM matter: because of anomalies these would have to have both positive and negative charge, and some of the soft masses would be negative. Also, the usual SM Yukawa couplings would need to be made invariant. There are a number of ways to proceed at this point, but a simple possibility is have SM fields $f$ and Higgses $H_{u, d}$ uncharged under the $U(1)$, but mixing with a vector like copy of all these fields $\left(F, F^{c}\right),\left(\tilde{H}_{u, d}, \tilde{H}_{u, d}^{c}\right)$, where the $\left(\tilde{H}_{u, d}\right)$ fields are completed into $(5+\overline{5})$ 's. These fields have masses of order $\mu$ and Yukawa couplings of the form $f \phi^{c} F^{c}$, so that after the $U(1)$ breaking, the light fields are an $O(1)$ mixture of the neutral fields and those of charge +1 , all of which have positive soft masses of order $\sim \mu^{2}$. Also, the $B_{\mu}$ term for the Higgses arises from the coupling $X H_{u} H_{d}$ which, as we have seen, is naturally of the same order of magnitude. Thus, we have found

$$
\tilde{m}^{2} \sim B_{\mu} \sim m^{2}
$$


At this level the gauginos and Higgsinos are massless. These in turn can be induced by integrating out physics at a higher scale $M_{*}$, perhaps near the GUT scale, which can give rise to couplings of the form

$$
\int d^{2} \theta \frac{X}{M_{*}} W^{\alpha} W^{\alpha}, \int d^{4} \theta \frac{X}{M_{*}} H_{u} H_{d}
$$

which generates

$$
M_{\tilde{g}} \sim \mu \sim \frac{\tilde{m}^{2}}{M_{*}} .
$$

Finally, the fermionic component of $Y$ is the Goldstino, which will be eaten by the gravitino to pick up a mass of order

$$
m_{3 / 2} \sim \frac{\tilde{m}^{2}}{M_{\mathrm{Pl}}}
$$

Meanwhile, without any particular suppression, the anomaly mediated contribution to the gaugino masses is of order $M_{\tilde{g}}^{\text {anom }} \sim \alpha /(4 \pi)\left(\tilde{m}^{2} / M_{\mathrm{Pl}}\right)$, so that even for $M_{*} \sim M_{\mathrm{Pl}}$ the contributions we have calculated dominate the gravitational ones. In these theories, the gravitino can naturally be comparable in mass or lighter than the gauginos and Higgsinos (the phenomenological consequences will be studied in sect. 5.3). If we wish $M_{*}$ to be above the GUT scale, we have $\tilde{m} \gtrsim 10^{9} \mathrm{GeV}$.

Note also that about this same scale $\tilde{m}$ we have all the extra vector-like matter, affecting the running of the SM couplings above $\tilde{m}$. In our simple example, we have added an equivalent of $14(5+\overline{5})$ 's at $\tilde{m}$. Even with the addition of all these new states, the SM couplings do not blow up beneath the GUT scale; however, the value of the GUT coupling can become so large that the rate from dimension-6 induced proton decay can become significant. The existence of extra vector-like matter charged under the SM is a feature of all direct mediation models, though the precise size of the sector is model-dependent. Therefore, we do generically expect the $p$-decay rates to be significantly enhanced in these models. Further discussion of proton decay in Split Supersymmetry will be given in section 7 .

\section{$5 \quad$ Dark Matter and Gravitinos in Split Supersymmetry}

In Split Supersymmetry the all-important mass scale of gauginos-higgsinos is decoupled from the electroweak scale and is set solely by the requirement of obtaining the correct dark matter abundance. It is therefore crucial to carefully compute the DM abundance in these theories.

A fundamentally new feature of Split Supersymmetry is that the masses of squarks and sleptons, as well as the gravitino, can be much heavier than a TeV. This can lead to new 
processes contributing to the DM abundance. In particular, the heavy scalars can decay or annihilate into gravitinos which hover, till they eventually decay into a final state including the LSP. Since this process occurs in addition to the canonical Lee-Weinberg annihilation, it typically results in an LSP number density exceeding the usual freezout abundance, and therefore in a lighter and more accessible LSP.

Another possibility is that the gravitino is lighter than all the gauginos-higgsinos, and therefore it is the LSP. This leads to interesting laboratory signatures in the decays of the NLSP and the gluino, summarized in sect. 5.3.

We will discriminate the various possible scenaria according to the gravitino mass which, as we have discussed in sect. 2, is an important parameter characterizing the theory. Let us start with some general properties of gravitinos. The gravitino decay width is given by

$$
\Gamma_{3 / 2}=\left(N_{g}+\frac{N_{m}}{12}\right) \frac{m_{3 / 2}^{3}}{32 \pi M_{\mathrm{Pl}}^{2}}=\left(\frac{m_{3 / 2}}{10^{5} \mathrm{GeV}}\right)^{3} 32 \mathrm{sec}^{-1}
$$

Here $N_{g}$ and $N_{m}$ are the numbers of available decay channels into gauge-gaugino and fermion-sfermion, respectively. In Split Supersymmetry, we will be mostly concerned with the case in which decay into squarks, sleptons and one Higgs doublet are not kinematically allowed, and thus $N_{g}=12, N_{m}=1$.

Since gravitinos in the early universe decay when $\Gamma_{3 / 2}=H$, the temperature after decay is given by

$$
T_{3 / 2}=\left[\frac{90 \Gamma_{3 / 2}^{2} M_{\mathrm{Pl}}^{2}}{\pi^{2} g_{*}\left(T_{3 / 2}\right)}\right]^{1 / 4}=\left[\frac{10.75}{g_{*}\left(T_{3 / 2}\right)}\right]^{1 / 4}\left(\frac{m_{3 / 2}}{10^{5} \mathrm{GeV}}\right)^{3 / 2} 6.8 \mathrm{MeV}
$$

where $g_{*}$ counts the effective number of degrees of freedom.

The gravitino number density in the early universe $n_{3 / 2}$, before decay, evolves according to the Boltzmann equation [17]

$$
\frac{d n_{3 / 2}}{d t}+3 H n_{3 / 2}=\left(\gamma_{\mathrm{sc}}+\gamma_{\mathrm{dec}}\right)\left(1-\frac{n_{3 / 2}}{n_{3 / 2}^{\mathrm{eq}}}\right)
$$

We assume that SM and supersymmetric particles (other than the gravitino) are in equilibrium. Two processes contribute to eq. (69): gravitino emission in scatterings and decay of thermalized supersymmetric particles into gravitinos.

The rate of gravitino production in scatterings $\gamma_{\mathrm{sc}}$ has been computed in supersymmetric QCD at finite temperature. Performing a hard thermal-loop resummation, where the contributions from soft momenta are regularized by considering an effective gluon propagator, 
the study of ref. [18] finds

$$
\begin{gathered}
\gamma_{\mathrm{sc}}=\frac{T^{6}}{M_{\mathrm{Pl}}^{2}} C_{\mathrm{sc}} \\
C_{\mathrm{sc}}=b\left(1+\frac{b^{2} M_{\tilde{g}}^{2}}{12 m_{3 / 2}^{2}}\right)\left\{1+0.48 n-0.74\left(1+\frac{n}{3}\right) \ln \left[b\left(1+\frac{n}{3}\right)\right]\right\} 8.4 \times 10^{-2} .
\end{gathered}
$$

Here $n$ is the number of effective quark-squark flavour multiplets ( $n=6$ if squarks are light, and $n=0$ in Split Supersymmetry with $T_{R}<\tilde{m}$ ), and $b(T)=2 \alpha_{s}(T) / \alpha_{s}\left(M_{\tilde{g}}\right)$ (in particular, $b(T) \simeq 1$ for $\left.T=10^{10} \mathrm{GeV}\right)$. The term proportional to the gluino mass in eq. (171) becomes important when $M_{*} \ll M_{\mathrm{Pl}}$, since the Goldstino coupling is enhanced. Notice that we have not included an analogous term proportional to the squark masses. Indeed, while the effective Goldstino coupling to gluinos is proportional to $M_{\tilde{g}} T / M_{S}^{2}$, the coupling to squarks is proportional to $\tilde{m}^{2} / M_{S}^{2}\left[19\right.$. Therefore, it will give a contribution $C_{\mathrm{sc}} \propto \tilde{m}^{4} /\left(m_{3 / 2} T\right)^{2}$. Even for Split Supersymmetry, where $\tilde{m} \gg M_{\tilde{g}}$, this contribution can be neglected, as it is subleading to the decay process we consider next.

Supersymmetric particles in thermal equilibrium can occasionally decay into gravitinos, with a rate

$$
\gamma_{\mathrm{dec}}=\sum_{i=1}^{N} \tilde{n}_{i}^{\mathrm{eq}} \frac{K_{1}\left(z_{i}\right)}{K_{2}\left(z_{i}\right)} \Gamma_{\mathrm{dec}}^{i}
$$

where the sum extends over all supersymmetric particles in the thermal bath with mass $\tilde{m}_{i}$, and $z_{i} \equiv \tilde{m}_{i} / T$. The ratio of Bessel functions $K_{1}\left(z_{i}\right) / K_{2}\left(z_{i}\right)$ describes the thermal average of the time dilatation factor $\tilde{m}_{i} / E$. Using, for simplicity, Maxwell-Boltzmann statistics for both scalars and fermions, the equilibrium density of the supersymmetric particles with 2 degrees of freedom is

$$
\tilde{n}_{i}^{\mathrm{eq}}=\frac{T^{3}}{\pi^{2}} z_{i}^{2} K_{2}\left(z_{i}\right)
$$

Finally, the decay width into gravitinos is the same for both gauginos and sfermions

$$
\Gamma_{\mathrm{dec}}^{i}=\frac{\tilde{m}_{i}^{5}}{48 \pi m_{3 / 2}^{2} M_{\mathrm{Pl}}^{2}} .
$$

It is convenient to rewrite eq. (69) in terms of $Y_{3 / 2} \equiv n_{3 / 2} / s$, the gravitino number density in units of entropy density $s$. We find the differential equation

$$
\frac{d Y_{3 / 2}}{d T}=-\frac{\left(\gamma_{\mathrm{sc}}+\gamma_{\mathrm{dec}}\right)}{H T s}\left(1-\frac{Y_{3 / 2}}{Y_{3 / 2}^{\mathrm{eq}}}\right),
$$

which can be easily integrated. The term proportional to $\gamma_{\mathrm{sc}}$ is dominated by the largest temperature $T_{R}$, to be interpreted as the reheat temperature after inflation or, in general, 
as the temperature after a significant entropy production. On the other hand, the term proportional to $\gamma_{\text {dec }}$ in eq. (175) is dominated by temperatures $T \sim \tilde{m}$. The solution is ${ }^{2}$

$$
\begin{gathered}
Y_{3 / 2}=Y_{3 / 2}^{\mathrm{eq}}\left[1-\exp \left(-\frac{Y_{3 / 2}^{\mathrm{sc}}+Y_{3 / 2}^{\mathrm{dec}}}{Y_{3 / 2}^{\mathrm{eq}}}\right)\right] \simeq \min \left(Y_{3 / 2}^{\mathrm{sc}}+Y_{3 / 2}^{\mathrm{dec}}, Y_{3 / 2}^{\mathrm{eq}}\right) \\
Y_{3 / 2}^{\mathrm{eq}}=\frac{135 \zeta(3)}{4 \pi^{4} g_{*}} \simeq 2 \times 10^{-3} \\
Y_{3 / 2}^{\mathrm{sc}}=\left.\frac{\gamma_{\mathrm{sc}}}{H s}\right|_{T=T_{R}}=\left(1+\frac{M_{\tilde{g}}^{2}}{12 m_{3 / 2}^{2}}\right)\left(\frac{T_{R}}{10^{10} \mathrm{GeV}}\right)\left[\frac{228.75}{g_{*}\left(T_{R}\right)}\right]^{3 / 2} 1.0 \times 10^{-12} \\
Y_{3 / 2}^{\mathrm{dec}}=\left.\frac{135}{4 \pi^{3}} \sum_{i=1}^{N} \frac{\Gamma_{\mathrm{dec}}^{i}}{g_{*} H}\right|_{T=\tilde{m}_{i}}=\left(\frac{\tilde{m}}{\mathrm{TeV}}\right)^{3}\left(\frac{\mathrm{GeV}}{m_{3 / 2}}\right)^{2}\left[\frac{228.75}{g_{*}(\tilde{m})}\right]^{3 / 2}\left(\frac{N}{46}\right) 1.2 \times 10^{-13} .
\end{gathered}
$$

The sum in eq. (79) is over all supersymmetric particles such that $m_{3 / 2}<\tilde{m}_{i}<T_{R}$. If only the heavy states of Split Supersymmetry satisfy this relation, then $N=46$.

In ordinary supersymmetry, the term $Y_{3 / 2}^{\text {dec }}$ is negligible, unless the gravitino is extremely light. In Split Supersymmetry with large $\tilde{m}$, the term $Y_{3 / 2}^{\text {dec }}$ can easily be the dominant source of gravitino production.

To proceed in our discussion, we consider different ranges of gravitino masses.

\subsection{Case $m_{3 / 2} \gtrsim 10^{5} \mathrm{GeV}$}

This is the case in which we have to require $F_{\phi} \ll m_{3 / 2}$, or else anomaly mediation gives an excessive contribution to gaugino masses. This can be done whenever supersymmetry is unbroken in the flat limit $\left(M_{\mathrm{Pl}} \rightarrow \infty\right)$, as discussed in sect. 3. In the range of $m_{3 / 2}$ considered in this section, the gravitino is too heavy to play any role in collider phenomenology. Also, gravitino decay occurs before the beginning of nucleosynthesis, see eq. (68), and therefore it is not harmful for the prediction of primordial-element abundances, in contrast to the ordinary case. However, the gravitino can significantly affect the neutralino relic abundance.

The lightest neutralino $\chi$ is stable and it decouples from the thermal bath at a freeze-out temperature $T_{f}$

$$
T_{f}=\frac{m_{\chi}}{x_{f}}, \quad x_{f}=28+\ln \left\{\frac{\mathrm{TeV}}{m_{\chi}} \frac{c}{10^{-2}}\left[\frac{86.25}{g_{*}\left(T_{f}\right)}\right]^{1 / 2}\right\} .
$$

\footnotetext{
${ }^{2}$ We are using $\int_{0}^{\infty} d z z^{3} K_{1}(z)=3 \pi / 2$.
} 
Here we have parametrized the non-relativistic $\chi$ annihilation cross section as

$$
\left\langle\sigma_{\chi} v_{\text {rel }}\right\rangle=\frac{c}{m_{\chi}^{2}}
$$

In Split Supersymmetry, $c=3 \times 10^{-3}$ for a mostly-higgsino $\chi$, and $c=1 \times 10^{-2}$ for a mostly $W$-ino $\chi$ (including the effects of coannihilation).

If $T_{3 / 2}>T_{f}$, all gravitino-decay products reach thermal and chemical equilibrium. The $\chi$ relic abundance will be unaffected by the gravitino decay process. We want to investigate the opposite case, in which the $\chi$ produced in gravitino decay are out of chemical equilibrium. This happens when

$$
T_{3 / 2}<T_{f} \Rightarrow \quad m_{3 / 2}<\left(\frac{m_{\chi}}{\mathrm{TeV}}\right)^{2 / 3}\left[\frac{g_{*}\left(T_{3 / 2}\right)}{86.25}\right]^{1 / 6} 4.3 \times 10^{7} \mathrm{GeV}
$$

The gravitino decay generates a population of $\chi$ with density

$$
\left.Y_{\chi}\left(T_{3 / 2}\right) \equiv \frac{n_{\chi}}{s}\right|_{T=T_{3 / 2}}=Y_{3 / 2}\left(\frac{T_{0}}{T_{3 / 2}}\right)^{3},
$$

where $Y_{3 / 2}$ is given in eq. (176). The factor $\left(T_{0} / T_{3 / 2}\right)^{3}$, where $T_{0}$ is the temperature of the thermal bath before gravitino decay, takes into account the entropy generated by the thermalized decay products. We can compute $T_{0}$ by balancing the energy density before and after the decay process, and thus by solving the equation

$$
\left(\frac{T_{0}}{T_{3 / 2}}\right)^{3}\left(\frac{T_{0}}{T_{3 / 2}}+\frac{4 Y_{3 / 2} m_{3 / 2}}{3 T_{3 / 2}}\right)=1 .
$$

For small $Y_{3 / 2}$, the solution is $\left(T_{0} / T_{3 / 2}\right)^{3} \simeq 1-Y_{3 / 2} m_{3 / 2} / T_{3 / 2}$ and therefore

$$
Y_{\chi}\left(T_{3 / 2}\right) \simeq Y_{3 / 2}\left(1-Y_{3 / 2} \frac{m_{3 / 2}}{T_{3 / 2}}\right) \quad \text { (radiation dominance) }
$$

The $\chi$ number density is equal to the gravitino number density before decay, and a small dilution factor due to entropy production appears in $Y_{\chi}\left(T_{3 / 2}\right)$.

For sufficiently large $T_{R}$ and low $T_{3 / 2}$ or for sufficiently large $\tilde{m}$ and small $m_{3 / 2}$, the universe eventually becomes gravitino-dominated. This happens when

$$
\begin{array}{cl}
T_{R} \gtrsim\left(\frac{m_{3 / 2}}{10^{5} \mathrm{GeV}}\right)^{1 / 2}\left[\frac{10.75}{g_{*}\left(T_{3 / 2}\right)}\right]^{1 / 4} 5 \times 10^{14} \mathrm{GeV} & \text { for } Y_{3 / 2}^{\mathrm{sc}}>Y_{3 / 2}^{\mathrm{dec}} \\
\tilde{m} \gtrsim\left(\frac{m_{3 / 2}}{10^{5} \mathrm{GeV}}\right)^{5 / 6}\left[\frac{10.75}{g_{*}\left(T_{3 / 2}\right)}\right]^{1 / 12} 2 \times 10^{8} \mathrm{GeV} & \text { for } Y_{3 / 2}^{\mathrm{dec}}>Y_{3 / 2}^{\mathrm{sc}}
\end{array}
$$


Then the solution of eq. (84) is $\left(T_{0} / T_{3 / 2}\right)^{3} \simeq 3 T_{3 / 2} /\left(4 Y_{3 / 2} m_{3 / 2}\right)$ and therefore

$$
Y_{\chi}\left(T_{3 / 2}\right) \simeq \frac{3 T_{3 / 2}}{4 m_{3 / 2}} \quad \text { (gravitino dominance) }
$$

Indeed, we could have directly obtained this result by noticing that, when the gravitino dominates the universe, all radiation is produced by the decay process and therefore $Y_{\chi}\left(T_{3 / 2}\right)=$ $\rho_{\text {rad }} /\left(s m_{3 / 2}\right)$, which coincides with eq. (88).

Notice that, if the universe becomes gravitino-dominated, the decay erases any primordial $\chi$ density. Region of parameter space which are ruled out by an excessive dark-matter density, as obtained by the standard thermal relic-density calculation, can now be reconsidered. Even if the gravitino never dominates the universe, its entropy production dilutes the primordial $Y_{\chi}$ by a factor $\left(T_{0} / T_{3 / 2}\right)^{3} \simeq 1-Y_{3 / 2} m_{3 / 2} / T_{3 / 2}$. Large entropy production leads to the potential problem of diluting any cosmic baryon asymmetry produced at temperatures larger than $T_{3 / 2}$. If the gravitino dominates the universe, we necessarily have to invoke a low-temperature mechanism for baryogenesis.

In balancing the energy density in eq. (84) we have neglected the $\chi$ contribution after gravitino decay. This is appropriate because neutralinos, although out of chemical equilibrium, are in kinetic equilibrium, since their rate for elastic scattering with the thermal bath is larger than the Hubble rate $\left(T_{3 / 2}^{3} \sigma_{\mathrm{el}}>H\right)$. This means that the $\chi$ will rapidly lose their kinetic energy, becoming non-relativistic, with an energy density $\rho_{\chi}=m_{\chi} n_{\chi}$. Since $n_{\chi}$ is equal to $n_{3 / 2}$ before decay, $\rho_{\chi}$ is about $m_{\chi} / m_{3 / 2}$ times smaller than the radiation energy density.

Since we are considering the case $T_{3 / 2}<T_{f}$, the particles $\chi$ produced by the gravitino decay are out of chemical equilibrium and satisfy the Boltzmann equation

$$
\frac{d n_{\chi}}{d t}+3 H n_{\chi}=-\left\langle\sigma_{\chi} v_{\text {rel }}\right\rangle n_{\chi}^{2}
$$

Using the variable $z=T / T_{3 / 2}$, eq. (89) can be written as

$$
\begin{gathered}
\frac{d Y_{\chi}^{-1}}{d z}=-\beta^{-1}, \\
\left.\beta \equiv \frac{H}{s\left\langle\sigma_{\chi} v_{\text {rel }}\right\rangle}\right|_{T=T_{3 / 2}}=\left(\frac{m_{\chi}}{\mathrm{TeV}}\right)^{2}\left(\frac{10^{5} \mathrm{GeV}}{m_{3 / 2}}\right)^{3 / 2}\left(\frac{10^{-2}}{c}\right)\left[\frac{86.25}{g_{*}\left(T_{3 / 2}\right)}\right]^{1 / 4} 8.5 \times 10^{-10} .
\end{gathered}
$$

The solution of this differential equation is

$$
Y_{\chi}^{-1}(\text { today })=Y_{\chi}^{-1}\left(T_{3 / 2}\right)+\beta^{-1}
$$




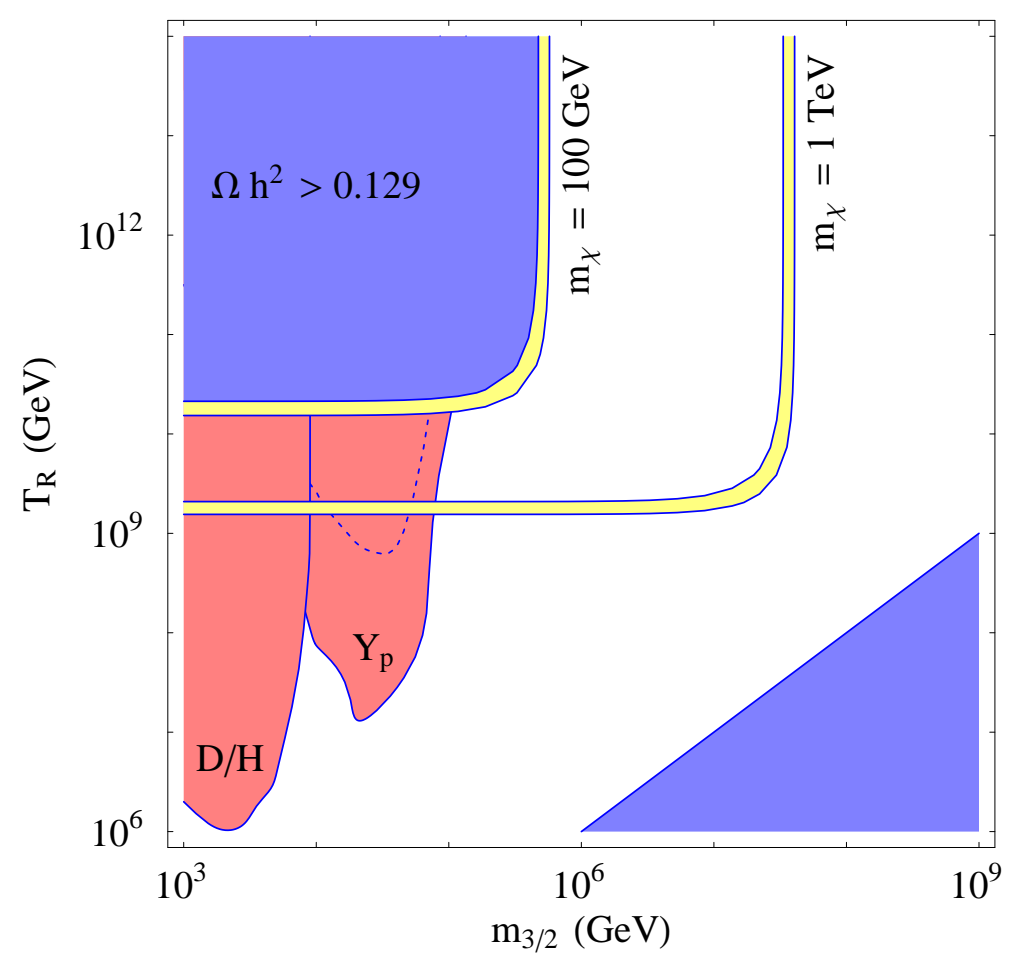

Figure 1: Case $\tilde{m}>T_{R}$. The yellow bands correspond to a neutralino density from gravitino decay $0.094<\Omega_{\chi} h^{2}<0.129$, for $c=3 \times 10^{-3}$, and $m_{\chi}=100 \mathrm{GeV}$ or $1 \mathrm{TeV}$. The region above the bands leads to an overdensity of dark matter. The red region is excluded by the nucleosynthesis constraints $D / H<3.6 \times 10^{-5}$ and $Y_{p}<0.249$. The dashed line corresponds to the weaker constraint $Y_{p}<0.253$. The region in the bottom-right corner is excluded by the requirement $T_{R}>m_{3 / 2}$.

This result can be easily understood. If $n_{\chi}\left\langle\sigma_{\chi} v_{\text {rel }}\right\rangle<H$ at $T=T_{3 / 2}\left(\right.$ i.e. $\left.Y_{\chi}\left(T_{3 / 2}\right)<\beta\right)$, then the neutralinos can never annihilate, and their number density is just diluted by expansion, and therefore $Y_{\chi}$ (today) $=Y_{\chi}\left(T_{3 / 2}\right)$. On the other hand, if the opposite inequality holds, the neutralinos will annihilate until their annihilation rate is equal to the expansion rate $H$, and thus $Y_{\chi}$ (today) $=\beta$.

Using today's ratio of critical density versus entropy density

$$
\left(\rho_{c} h^{-2} / s\right)_{\text {today }}=3.5 \times 10^{-12} \mathrm{TeV},
$$

we obtain

$$
\Omega_{\chi} h^{2}=\left.\frac{m_{\chi} Y_{\chi}}{\rho_{c} h^{-2} / s}\right|_{\text {today }}=\left(\frac{m_{\chi}}{\mathrm{TeV}}\right)\left[Y_{\chi}^{-1}\left(T_{3 / 2}\right)+\beta^{-1}\right]^{-1} 2.8 \times 10^{11},
$$

where $Y_{\chi}^{-1}\left(T_{3 / 2}\right)$ is given by eq. (83). 
We first consider the case $\tilde{m}>T_{R}$, in which $Y_{3 / 2}^{\text {dec }}$ can be neglected. In fig. 1 we show the parameter region for which the non-thermal population of neutralinos from gravitino decay can account for the required dark matter density in a 2- $\sigma$ range $0.094<\Omega_{\chi} h^{2}<0.129$ [20]. The result shown in fig. \is well explained by considering two limiting cases. For $Y_{\chi}\left(T_{3 / 2}\right)<$ $\beta$, when $\chi$ annihilation is irrelevant, we find

$$
\begin{gathered}
\Omega_{\chi} h^{2}=\left(\frac{m_{\chi}}{\mathrm{TeV}}\right)\left(\frac{T_{R}}{10^{10} \mathrm{GeV}}\right) 0.3, \\
\Omega_{\chi} h^{2}<0.129 \Rightarrow \quad T_{R}<\left(\frac{\mathrm{TeV}}{m_{\chi}}\right) 5 \times 10^{9} \mathrm{GeV},
\end{gathered}
$$

which describes the horizontal branches of the curves in fig. (1) Notice that eq. (95)) depends neither on the microphysics $\chi$ interactions nor on the gravitino mass. For an appropriate value of $T_{R}$, we can explain the correct dark-matter density for neutralino masses which are inadequate to give the right thermal abundance.

On the other hand, for $Y_{\chi}\left(T_{3 / 2}\right)>\beta$ we find

$$
\begin{gathered}
\Omega_{\chi} h^{2}=\left(\frac{m_{\chi}}{\mathrm{TeV}}\right)^{3}\left(\frac{10^{5} \mathrm{GeV}}{m_{3 / 2}}\right)^{3 / 2}\left(\frac{10^{-2}}{c}\right)\left[\frac{86.25}{g_{*}\left(T_{3 / 2}\right)}\right]^{1 / 4} 2 \times 10^{2}, \\
\Omega_{\chi} h^{2}<0.129 \Rightarrow \quad m_{3 / 2}>\left(\frac{m_{\chi}}{\mathrm{TeV}}\right)^{2}\left(\frac{10^{-2}}{c}\right)^{2 / 3}\left[\frac{86.25}{g_{*}\left(T_{3 / 2}\right)}\right]^{1 / 6} 2 \times 10^{7} \mathrm{GeV},
\end{gathered}
$$

which describes the vertical branches of the curves in fig. 1. This result depends on $m_{3 / 2}$ and on the neutralino annihilation cross section, but it is independent on $T_{R}$ and on the initial gravitino density (as long as it large enough to satisfy $Y_{\chi}\left(T_{3 / 2}\right)>\beta$ ). An appropriate $\chi$ relic density can be found for interesting values of $m_{3 / 2}$, which are consistent with the requirement $T_{3 / 2}<T_{\mathrm{dec}}$, see eq. (82).

Next, we consider the mass range

$$
T_{R}>\tilde{m}>\left(\frac{T_{R}}{10^{10} \mathrm{GeV}}\right)^{1 / 3}\left(\frac{m_{3 / 2}}{10^{5} \mathrm{GeV}}\right)^{2 / 3} 4 \times 10^{6} \mathrm{GeV},
$$

in which $Y_{3 / 2}^{\text {dec }}>Y_{3 / 2}^{\text {sc }}$, and therefore the gravitino density is independent of $T_{R}$.

In this case, the parameter region in which neutralinos from gravitino decay can account for the dark matter in shown in fig. 2. Again, the two branches of the curves can be simply understood. The vertical branch is given by eqs. (97)-(98), while the oblique branch corresponds to $Y_{\chi}$ (today) $\simeq Y_{3 / 2}^{\text {dec }}$ and therefore

$$
\Omega_{\chi} h^{2}=\left(\frac{m_{\chi}}{\mathrm{TeV}}\right)\left(\frac{\tilde{m}}{10^{7} \mathrm{GeV}}\right)^{3}\left(\frac{10^{5} \mathrm{GeV}}{m_{3 / 2}}\right)^{2}\left(\frac{N}{46}\right) 3
$$




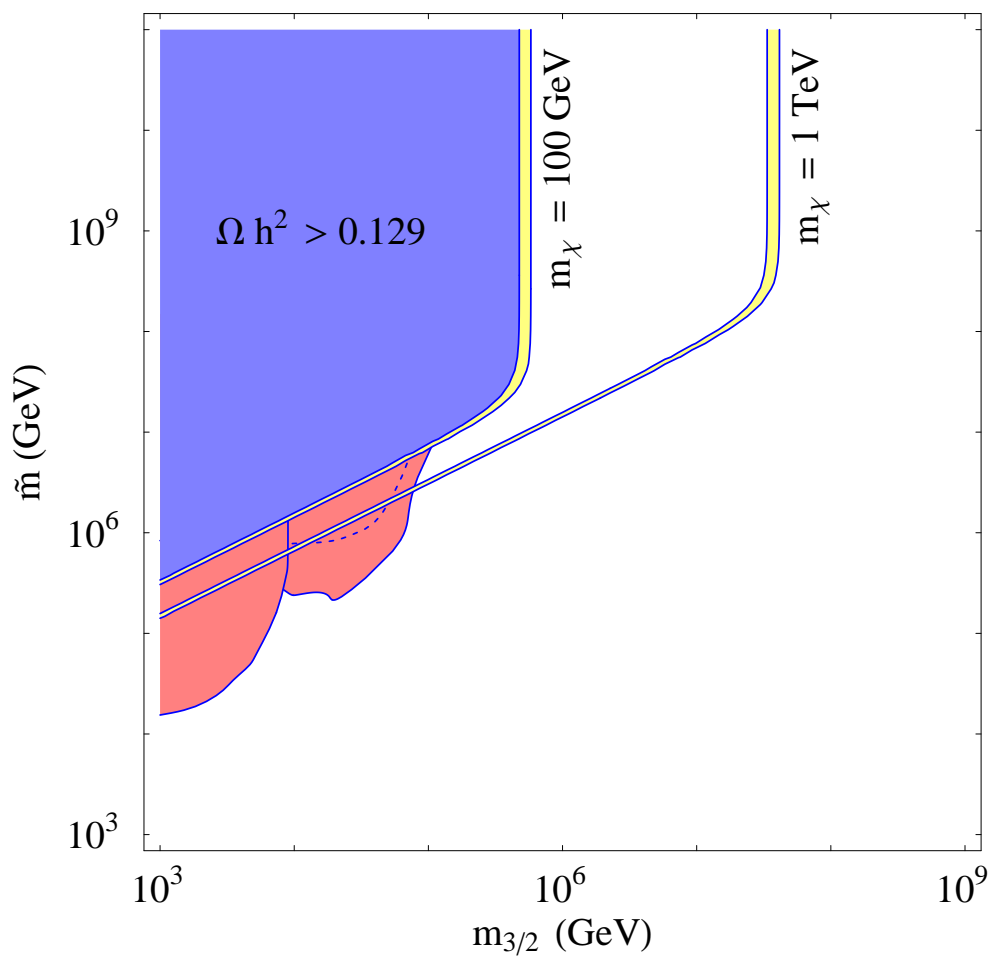

Figure 2: Case $T_{R}>\tilde{m}>\left(T_{R} / 10^{10} \mathrm{GeV}\right)^{1 / 3}\left(m_{3 / 2} / 10^{5} \mathrm{GeV}\right)^{2 / 3} 4 \times 10^{6} \mathrm{GeV}$. The yellow bands correspond to a neutralino density from gravitino decay $0.094<\Omega_{\chi} h^{2}<0.129$, for $c=3 \times 10^{-3}$, and $m_{\chi}=100 \mathrm{GeV}$ or $1 \mathrm{TeV}$. The region above the bands leads to an overdensity of dark matter. The red region is excluded by the nucleosynthesis constraints $D / H<3.6 \times 10^{-5}$ and $Y_{p}<0.249$. The dashed line corresponds to the weaker constraint $Y_{p}<0.253$.

$$
\Omega_{\chi} h^{2}<0.129 \Rightarrow \quad \tilde{m}<\left(\frac{\mathrm{TeV}}{m_{\chi}}\right)^{1 / 3}\left(\frac{m_{3 / 2}}{10^{5} \mathrm{GeV}}\right)^{2 / 3}\left(\frac{46}{N}\right)^{1 / 3} 3 \times 10^{6} \mathrm{GeV} .
$$

It is interesting that, under the condition in eq. (99), $\Omega_{\chi}$ from gravitino decay is completely determined in terms of the mass parameters of the theory and it does not depend on the cosmological initial condition parametrized by $T_{R}$.

Figures 11 and 2 illustrate how gravitino decay can lead to the correct dark-matter neutralino density under conditions in which the usual thermal abundance is insufficient. This has important implications for phenomenological analyses of Split Supersymmetry. Indeed, we recall that the value of the dark-matter density plays a crucial role in Split Supersymmetry, since it offers a rationale to relate the gaugino-higgsino masses to the weak scale. Cases which were excluded by consideration of thermal relic abundances (as an LSP higgsino lighter than $1 \mathrm{TeV}$ or an LSP W-ino lighter than $2 \mathrm{TeV}$ [8]) can be consistent with the observed amount of dark matter, for appropriate values of $m_{3 / 2}$. 
The prospects for direct and indirect dark-matter detection are also affected. Neutralinos with large annihilation cross sections can properly account for the dark matter, because of gravitino decay. Since squarks are heavy, the only contribution to spin-independent neutralino-nuclei interactions comes from Higgs-boson exchange [21]. The $\chi$ scattering cross section off a proton is given by

$$
\sigma_{p}=\frac{8}{\pi}\left[\frac{G_{\mathrm{F}} M_{W} m_{p} \mu_{\chi}}{9 m_{H}^{2}}\left(2+7 \sum_{q=u, d, s} f_{q}^{(p)}\right) \gamma\right]^{2}=\left(\frac{115 \mathrm{GeV}}{m_{H}}\right)^{4} \gamma^{2} 5.4 \times 10^{-43} \mathrm{~cm}^{2}
$$

where 22] $f_{u}^{(p)}=0.023, f_{d}^{(p)}=0.034, f_{s}^{(p)}=0.14$ and $\gamma$ measures the Higgs coupling with the LSP neutralino

$$
\gamma=\frac{1}{g}\left(\tilde{g}_{u} N_{\chi 2} N_{\chi 4}-\tilde{g}_{d} N_{\chi 2} N_{\chi 3}-\tilde{g}_{u}^{\prime} N_{\chi 1} N_{\chi 4}+\tilde{g}_{d}^{\prime} N_{\chi 1} N_{\chi 3}\right) .
$$

Here $N_{\chi i}$ are the lightest neutralino components in standard notations and $\tilde{g}_{u, d}, \tilde{g}_{u, d}^{\prime}$ are the higgsino couplings (see sect. 6). The coefficient $\gamma$ vanishes if $\chi$ is a pure Higgsino or gaugino and in the limit $\mu, M_{1,2} \gg M_{Z}$ becomes (assuming $M_{2}>M_{1}$ )

$$
\gamma=\cos \theta_{W} M_{Z} \frac{\left(\tilde{g}_{d}^{\prime 2}+\tilde{g}_{u}^{\prime 2}\right) M_{1}+2 \tilde{g}_{u}^{\prime} \tilde{g}_{d}^{\prime} \mu}{g^{2}\left(\mu^{2}-M_{1}^{2}\right)}+\mathcal{O}\left(\frac{M_{Z}^{2}}{M_{1,2}^{2}}, \frac{M_{Z}^{2}}{\mu^{2}}\right) .
$$

The maximum value of $\gamma$ is reached when $M_{1} \simeq \mu$. In this degenerate limit, eq. (104) is no longer valid, and it is replaced by

$$
\gamma=\frac{\tilde{g}_{u}^{\prime}+\tilde{g}_{d}^{\prime}}{2 \sqrt{2} g}+\cos \theta_{W} \frac{M_{Z}}{8 g^{2}}\left[2 \frac{\left(\tilde{g}_{d}+\tilde{g}_{u}\right)^{2}}{M_{2}-\mu}-\frac{\left(\tilde{g}_{d}^{\prime}-\tilde{g}_{u}^{\prime}\right)^{2}}{\mu}\right]+\mathcal{O}\left(\frac{M_{Z}^{2}}{M_{1,2}^{2}}, \frac{M_{Z}^{2}}{\mu^{2}}, \frac{M_{1}-\mu}{\mu}\right) .
$$

The second term in the expansion is actually numerically important because it is enhanced with respect to the leading term by a coefficient $1 / \tan \theta_{W}$. Notice that the maximal value of $\gamma$, given by eq. (105), is actually achieved in a large portion of the parameter space of Split Supersymmetry, leading to an appropriate dark-matter thermal abundance [8]. This is because an efficient annihilation rate approximately requires $M_{1} \simeq \mu$. In fig. 3 we show the spin-independent $\chi$ scattering cross section off protons, without requiring any constraints on $\Omega_{\chi}$ and therefore assuming that gravitino decay accounts for the correct value of $\Omega_{\chi}$. The rate is within the reach of future experiments, which can reach $10^{-44}-10^{-45} \mathrm{~cm}^{2}$ for $m_{\chi}<1 \mathrm{TeV}$.

We also want to stress that the gravitino decay process does not weaken the link between neutralino masses and the weak scale. This link is based on the upper bound on the $\chi$ mass derived by the requirement that the thermal relic abundance (for $s$-wave annihilation)

$$
\left(\Omega_{\chi} h^{2}\right)_{\mathrm{th}}=\left(\frac{m_{\chi}}{\mathrm{TeV}}\right)^{2}\left(\frac{10^{-2}}{c}\right)\left(\frac{x_{f}}{28}\right)\left[\frac{86.25}{g_{*}\left(T_{f}\right)}\right]^{1 / 2} 3 \times 10^{-2}
$$




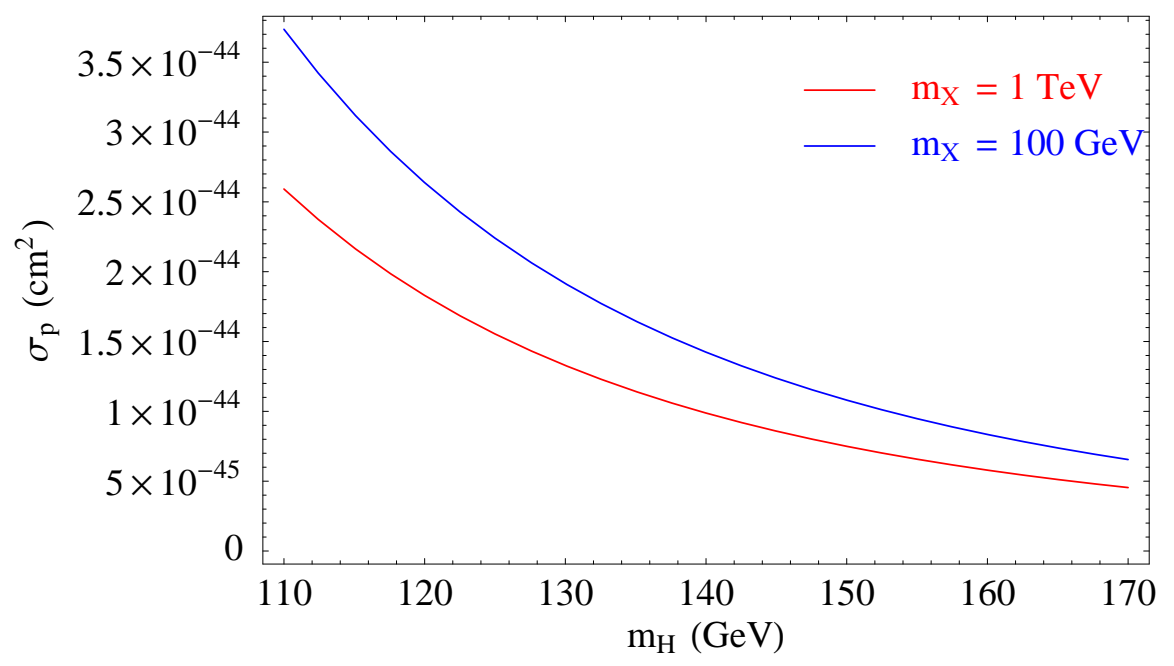

Figure 3: The maximum value of the spin-independent $\chi$ scattering cross section off protons, as a function of the Higgs mass $m_{H}$ and for two values of $m_{\chi}$. We have assumed eq. (121) at the chargino mass scale, and taken $\tan \beta=10$. No constraints on $\Omega_{\chi}$ are used, assuming that gravitino decay accounts for the correct value of $\Omega_{\chi}$.

does not exceed the observed value. The requirement $\left(\Omega_{\chi} h^{2}\right)_{\text {th }}<0.129$ gives

$$
m_{\chi}<\left(\frac{c}{10^{-2}}\right)^{1 / 2}\left(\frac{28}{x_{f}}\right)^{1 / 2}\left[\frac{g_{*}\left(T_{f}\right)}{86.25}\right]^{1 / 4} 2 \mathrm{TeV}
$$

where $c$ and $x_{f}$ are defined in eqs. (80)-(81). The value of the non-thermal $\Omega_{\chi}$ computed in this section has to be added to the thermal result in eq. (106) and therefore it can only lead to an upper bound on $m_{\chi}$ which is stronger than eq. (107). Even in the case in which the gravitino dominates the universe and dilutes the initial $\chi$ abundance, the upper bound on $m_{\chi}$ is tightened. Indeed, for a gravitino-dominated universe, eq. (97) applies. Then we can interpret eq. (98) as an upper bound on $m_{\chi}$, as a function of $m_{3 / 2}$. This bound becomes less stringent as $m_{3 / 2}$ grows, but a maximum allowed value of $m_{3 / 2}$ is determined by the condition $T_{3 / 2}<T_{f}$ in eq. (82). For the value of $m_{3 / 2}$ corresponding to $T_{3 / 2}=T_{f}$ we find an upper bound on $m_{\chi}$ which coincides with eq. (107), while for other values of $m_{3 / 2}$ the bound is stronger. The only exception in which the neutralino mass could be much larger than the value determined by eq. (107) occurs in the extreme case when $T_{R}$ is of the order of $T_{f}$ [23].

\subsection{Case $m_{\chi}<m_{3 / 2} \lesssim 10^{5} \mathrm{GeV}$}

In this $m_{3 / 2}$ range, the anomaly-mediated contributions to soft masses are acceptable and they can actually account for the entire values of gaugino masses, since they give [24] $M_{1} \simeq$ 
$m_{3 / 2} / 100, M_{2} \simeq m_{3 / 2} / 300$ and $M_{\tilde{g}} \simeq m_{3 / 2} / 40$. The collider phenomenology is then analogous to the one of anomaly mediation, but with two very significant differences. Squarks and sleptons can be much heavier than gauginos and higgsinos, depending on the value of $M_{*}$. The parameter $\mu$ is not determined by electroweak symmetry breaking to be larger than $M_{2}$ and therefore the lightest supersymmetric particle could be a higgsino, or have a higgsino component.

The gravitino lifetime $\tau_{3 / 2}$ is long enough to affect the nucleosynthesis predictions. For the values of $\tau_{3 / 2}$ considered in this section, the strongest bounds come from hadronic gravitino decays [25, 26, 27], while decays into photons [28, 29] play no role. This is because, injected photons have a high probability of scattering off background electrons and photons, still very numerous at this stage of nucleosynthesis, and they thermalize before having the chance of photo-dissociating recently-created nuclei. But, since we are considering the case $m_{3 / 2}>M_{\tilde{g}}$, we expect that the gravitinos decay into hadrons with a branching fraction of order unity, and therefore we turn to discuss the nucleosynthesis limits from hadronic decay modes.

In the region $10 \mathrm{TeV} \lesssim m_{3 / 2} \lesssim 100 \mathrm{TeV}\left(30 \mathrm{sec} \gtrsim \tau_{3 / 2} \gtrsim 3 \times 10^{-2} \mathrm{sec}\right)$, quarks and gluons produced by gravitino decay quickly hadronize, and the hadrons lose their kinetic energy by scattering off background electrons and photons without causing hadro-dissociation of nuclei. However, there can still be an effect on nucleosynthesis, because the slowed-down hadrons interact with ambient nucleons, converting neutrons into protons and viceversa. This will change the ratio $n / p$, which was fixed after weak interactions have frozen out. Since protons are more abundant than neutrons, the gravitino decay products will increase the ratio $n / p$ and therefore the predicted ${ }^{4} \mathrm{He}$ fraction. Using the results presented in ref. [27, we set the bounds on $T_{R}$ in fig. 1 and on $\tilde{m}$ in fig. 2 which correspond to a $95 \% \mathrm{CL}$ limit on the ${ }^{4} \mathrm{He}$ fraction $Y_{p}<0.249$ [30]. To illustrate the strong sensitivity of these bounds on $Y_{p}$, we also show in figs. 1 and 2 the weaker limit corresponding to $Y_{p}<0.253$, obtained from the study in ref. 31.

In the region $1 \mathrm{TeV} \lesssim m_{3 / 2} \lesssim 10 \mathrm{TeV}\left(3000 \mathrm{sec} \gtrsim \tau_{3 / 2} \gtrsim 30 \mathrm{sec}\right)$, injected hadrons can directly destroy nuclei. In particular, hadro-dissociation of ${ }^{4} \mathrm{He}$ leads to an overabundance of deuterium. Using a 95\% CL limit $D / H<3.6 \times 10^{-5}$ [32] and the calculation of ref. [27], we obtain the bounds shown in figs. 1 and 2 . Notice that in our case photo-dissociation of ${ }^{4} \mathrm{He}$ is irrelevant, since it starts to be effective only after about $10^{5}$ seconds [29]. Overproduction of ${ }^{6} \mathrm{Li}$ by hadro-dissociation starts to become competitive with the bounds from deuterium only for $m_{3 / 2}$ close to $1 \mathrm{TeV}$.

From the results shown in figs. 1]and 2, it is apparent that nucleosynthesis provides strong 
constraints on $T_{R}$ and $\tilde{m}$. In particular, from this bound we infer that, in the range of $m_{3 / 2}$ considered in this section, the gravitino decay process cannot generate a neutralino density sufficient to explain the dark matter. In this case, the appropriate value of $\Omega_{\chi} h^{2}$ has to be generated by the neutralino thermal relic abundance.

\subsection{Case $m_{3 / 2}<m_{\chi}$}

In this mass region, the gravitino is the LSP. In the absence of $R$-parity breaking, it is stable and its contribution to the present energy density is

$$
\Omega_{3 / 2} h^{2}=\frac{m_{3 / 2} Y_{3 / 2}}{\left(\rho_{c} h^{-2} / s\right)_{\text {today }}}
$$

where $Y_{3 / 2}$ is given in eq. (76) and today's ratio of critical density to entropy is given in eq. (93). The abundance $Y_{3 / 2}$ has three distinct behaviors. If $T_{R}$ or $\tilde{m}$ are large enough, gravitinos go in equilibrium and

$$
\begin{aligned}
& \Omega_{3 / 2} h^{2}=\left(\frac{m_{3 / 2}}{\mathrm{keV}}\right)\left(\frac{228.75}{g_{*}}\right) 0.5 \text { for } Y_{3 / 2}=Y_{3 / 2}^{\mathrm{eq}} \\
& \Omega_{3 / 2} h^{2}<0.129 \Rightarrow \quad m_{3 / 2}<\left(\frac{g_{*}}{228.75}\right) 0.3 \mathrm{keV}
\end{aligned}
$$

Here $g_{*}$ has to be computed at the gravitino decoupling temperature. If eq. (99) holds, than the decay processes dominate the gravitino density and

$$
\begin{gathered}
\Omega_{3 / 2} h^{2}=\left(\frac{\tilde{m}}{\mathrm{TeV}}\right)^{3}\left(\frac{\mathrm{MeV}}{m_{3 / 2}}\right)\left[\frac{228.75}{g_{*}(\tilde{m})}\right]^{3 / 2}\left(\frac{N}{46}\right) 3 \times 10^{-2} \text { for } Y_{3 / 2}=Y_{3 / 2}^{\mathrm{dec}}, \\
\Omega_{3 / 2} h^{2}<0.129 \Rightarrow \quad \tilde{m}<\left(\frac{m_{3 / 2}}{\mathrm{MeV}}\right)^{1 / 3}\left[\frac{g_{*}(\tilde{m})}{228.75}\right]^{1 / 2}\left(\frac{46}{N}\right)^{1 / 3} 2 \mathrm{TeV} .
\end{gathered}
$$

Otherwise, the scattering processes dominate the gravitino production and

$$
\begin{gathered}
\Omega_{3 / 2} h^{2}=\left(\frac{\mathrm{MeV}}{m_{3 / 2}}\right)\left(\frac{M_{\tilde{g}}}{\mathrm{TeV}}\right)^{2}\left(\frac{T_{R}}{10^{10} \mathrm{GeV}}\right)\left[\frac{228.75}{g_{*}\left(T_{R}\right)}\right]^{3 / 2} 2 \times 10^{4} \quad \text { for } Y_{3 / 2}=Y_{3 / 2}^{\mathrm{sc}}, \\
\Omega_{3 / 2} h^{2}<0.129 \Rightarrow \quad T_{R}<\left(\frac{m_{3 / 2}}{\mathrm{MeV}}\right)\left(\frac{\mathrm{TeV}}{M_{\tilde{g}}}\right)^{2}\left[\frac{g_{*}\left(T_{R}\right)}{228.75}\right]^{3 / 2} 5 \times 10^{4} \mathrm{GeV} .
\end{gathered}
$$

Finally the decay of the next-to-lightest supersymmetric particle (NLSP) provides an additional source of relic gravitinos,

$$
\Omega_{3 / 2} h^{2}=\frac{m_{3 / 2}}{m_{\chi}}\left(\Omega_{\chi} h^{2}\right)_{\text {th }} \quad \text { for NLSP decay, }
$$




$$
\Omega_{3 / 2} h^{2}<0.129 \Rightarrow \quad m_{3 / 2}<\left(\frac{\mathrm{TeV}}{m_{\chi}}\right)\left(\frac{c}{10^{-2}}\right)\left(\frac{28}{x_{f}}\right)\left[\frac{g_{*}\left(T_{f}\right)}{86.25}\right]^{1 / 2} 4 \times 10^{3} \mathrm{GeV} .
$$

We have used the thermal abundance of $\operatorname{NLSP}\left(\Omega_{\chi} h^{2}\right)_{\text {th }}$ given by eq. (106). Around the inflationary epoch, other sources of non-thermal gravitino production can be present [33], like the effect of the classical gravitational background on the vacuum state during the evolution of the universe. We will ignore here these mechanisms, which are more model-dependent.

The limits from eqs. (112) and (114) become very stringent for small gravitino masses. Of course, whenever the inequalities are nearly saturated, then gravitinos can account for the dark matter. However if gravitinos, rather than neutralinos, form the dark matter, then we lose the connection between the higgsino-gaugino masses and the weak scale, which is a critical ingredient of Split Supersymmetry.

Actually an upper bound on $m_{\chi}$ persists, even when the gravitino is the dark matter particle. Indeed, $\Omega_{3 / 2}$ from NLSP decays scales like $\Omega_{3 / 2} \propto m_{3 / 2} m_{\chi}$, see eq. (115). Large values of $m_{\chi}$ require small values of $m_{3 / 2}$ to avoid an excessive gravitino dark-matter density. On the other hand, the contribution to the gravitino energy density from thermal processes scales like $\Omega_{3 / 2} \propto m_{3 / 2}^{-1}$, see eqs. (111) and (113), and a small $m_{3 / 2}$ gives an excessive $\Omega_{3 / 2}$. Depending on the value of $T_{R}$ and $\tilde{m}$, the upper bound on $m_{\chi}$ can be very restrictive. However, it is not sufficient to unescapably tie the gaugino-higgsino masses to the weak scale. For instance, in the extreme case $\tilde{m}>T_{R} \sim M_{\tilde{g}} \sim m_{\chi}$, a dark-matter gravitino of about $10 \mathrm{GeV}$ is compatible with $m_{\chi}$ as large as $100 \mathrm{TeV}$.

The NLSP lifetime

$$
\tau_{\chi}=\frac{48 \pi M_{\mathrm{Pl}}^{2} m_{3 / 2}^{2}}{m_{\chi}^{5}}=\left(\frac{m_{3 / 2}}{\mathrm{MeV}}\right)^{2}\left(\frac{\mathrm{TeV}}{m_{\chi}}\right)^{5} 6 \times 10^{-7} \mathrm{sec}
$$

has a strong dependence on $m_{\chi}$ and $m_{3 / 2}$, but the decay can occur at late times and affect nucleosynthesis predictions and the cosmic microwave background. Large ranges of parameters are excluded [27, 29, 34. In particular, the case of a weak-scale gravitino is ruled out as a dark-matter candidate for a neutralino NLSP [35].

\subsection{Collider Phenomenology for Gravitino LSP}

The collider phenomenology with a gravitino LSP can be quite different than in ordinary low-energy supersymmetry, if the NLSP decay occurs within measurable distances. The NLSP can decay into $\gamma, Z$, or $H$ and missing energy. The signals will be similar to those of gauge mediation [36], in the limit of heavy scalars. 
A peculiarity of Split Supersymmetry, in the light-gravitino scenario, is represented by the gluino. Although the gluino is not the LSP, its dominant decay mode could be into gravitinos. Indeed, the decay width into gravitinos $\tilde{G}$ is

$$
\Gamma(\tilde{g} \rightarrow g \tilde{G})=\frac{M_{\tilde{g}}^{5}}{16 \pi M_{\mathrm{Pl}}^{2} m_{3 / 2}^{2}}\left(1-\frac{m_{3 / 2}^{2}}{M_{\tilde{g}}^{2}}\right)^{3}\left(1+3 \frac{m_{3 / 2}^{2}}{M_{\tilde{g}}^{2}}\right) .
$$

The ordinary gluino decay is suppressed by the large value of $\tilde{m}$ and by the 3-body phase space,

$$
\Gamma(\tilde{g} \rightarrow q \bar{q} \chi)=\frac{\alpha \alpha_{s} \mathcal{N} M_{\tilde{g}}^{5}}{192 \pi \sin ^{2} \theta_{W} \tilde{m}^{4}} .
$$

Here $\mathcal{N}$ is a factor which appropriately counts all decay channels into different quarks and charginos or neutralinos. For $m_{3 / 2} \simeq \tilde{m}^{2} / M_{\mathrm{Pl}}$ and $m_{3 / 2}<M_{\tilde{g}}$, the dominant decay mode is $\tilde{g} \rightarrow g \tilde{G}$, and each gluino leads to a single jet with characteristic energy distribution. The same final state could be mimicked, in a theory with heavy gravitino, by the radiative process $\tilde{g} \rightarrow g \chi$, where $\chi$ is the neutralino LSP. Such process is induced by the one-loop generated operator $\bar{\chi} \sigma^{\mu \nu} \gamma_{5} \tilde{g}^{a} G_{\mu \nu}^{a}$, where $\gamma_{5}$ arises because of the Majorana nature of $\tilde{g}$ and $\chi$. This operator is $\mathrm{C}$ (and $\mathrm{P}$ ) odd, and therefore it is not generated when left and right squarks are mass degenerate. Therefore, we expect a certain suppression. When the decay mode $\tilde{g} \rightarrow g \tilde{G}$ dominates over the tree-level 3-body process, it is also dominant with respect to the one-loop 2-body decay. A dominant gluino decay into a single jet and missing energy is a very distinctive signature of Split Supersymmetry with direct mediation.

\section{CP Violation and Electric Dipole Moments}

Split Supersymmetry resolves the difficulties with flavour and CP violation, encountered in generic supersymmetric models, because squarks and sleptons are taken to be very heavy, with masses of the order of $\tilde{m}$. Nevertheless, the effective theory below $\tilde{m}$ still contains physical CP-violating phases.

Consider the interaction Lagrangian of higgsinos $\left(\tilde{H}_{u, d}\right), \mathrm{W}$-ino $(\tilde{W})$ and B-ino $(\tilde{B})$ with the Higgs doublet $H$

$$
\begin{aligned}
-\mathcal{L} & =\frac{M_{2}}{2} \tilde{W}^{a} \tilde{W}^{a}+\frac{M_{1}}{2} \tilde{B} \tilde{B}+\mu \tilde{H}_{u}^{T} \epsilon \tilde{H}_{d} \\
& +\frac{H^{\dagger}}{\sqrt{2}}\left(\tilde{g}_{u} \sigma^{a} \tilde{W}^{a}+\tilde{g}_{u}^{\prime} \tilde{B}\right) \tilde{H}_{u}+\frac{H^{T} \epsilon}{\sqrt{2}}\left(-\tilde{g}_{d} \sigma^{a} \tilde{W}^{a}+\tilde{g}_{d}^{\prime} \tilde{B}\right) \tilde{H}_{d}+\text { h.c. },
\end{aligned}
$$

where $\epsilon=i \sigma_{2}$ and, at the scale $\tilde{m}$,

$$
\tilde{g}_{u}^{(\prime)}=g^{(\prime)} \sin \beta, \quad \tilde{g}_{d}^{(\prime)}=g^{(\prime)} \cos \beta .
$$


The gaugino and higgsino masses $M_{1,2}$ and $\mu$ are, in general, complex parameters. They can all be made real by a field redefinition. However, the phase redefinition of gauginos and higgsinos will induce two independent phases in the couplings $\tilde{g}_{u}$ and $\tilde{g}_{d}$ (and analogously in $\left.\tilde{g}_{u, d}^{\prime}\right)$. There is still the freedom to transform $H \rightarrow e^{i \alpha} H, \tilde{H}_{u} \rightarrow e^{i \beta} \tilde{H}_{u}, \tilde{H}_{d} \rightarrow e^{-i \beta} \tilde{H}_{d}$, without reintroducing phases in $M_{1,2}$ and $\mu$. However, this will induce the transformations $\tilde{g}_{u} \rightarrow e^{i(\beta-\alpha)} \tilde{g}_{u}, \tilde{g}_{d} \rightarrow e^{i(\alpha-\beta)} \tilde{g}_{d}$, and therefore only one of the two independent phases can be eliminated. In conclusion, the two quantities $\operatorname{Im}\left(\tilde{g}_{u}^{*} \tilde{g}_{d}^{*} M_{2} \mu\right)$ and $\operatorname{Im}\left(\tilde{g}_{u}^{\prime *} \tilde{g}_{d}^{\prime *} M_{1} \mu\right)$ are invariant under any field-phase redefinition and therefore correspond to physical CP-violating effects ${ }^{3}$. In most models of supersymmetry breaking, the phases of the different gaugino masses $M_{1,2}$ are equal, and therefore there is actually a single CP-violating invariant. Notice also that the invariants vanish in the limit in which $M_{1,2}$ and $\mu$ have opposite phases (in the notation of eq. (120) above) or when either $\tilde{g}_{u}$ or $\tilde{g}_{d}$ are absent (as it is approximately true in the limit of large $\tan \beta$ ).

The new phases are in the chargino-neutralino sector which does not couple at tree level to quarks and leptons, and therefore CP violation in processes involving SM fermions occurs only at two loops. The most interesting effect appears in the fermion electric dipole moments (EDM). These are induced by the effective operator $\bar{f} \sigma_{\mu \nu} \gamma_{5} f F^{\mu \nu}$, where $f$ is a generic fermion ( $f=e$ for the electron EDM, and $f=u, d, s$ for the neutron EDM). As gluinos carry no phases, there are no contributions from either chromomagnetic or 3-gluon operators [37] at the leading order.

The fermion EDM is generated by the two-loop diagrams shown in fig. 4. Other diagrams can also be constructed, by replacing the $\gamma$ and $H$ internal lines with various combinations of Higgs and weak gauge bosons [38]. However, the diagrams shown in fig. 4 form a gaugeinvariant set which is expected to give the leading contribution. In particular, notice that diagrams with $Z$ bosons give a contribution to the electron EDM which is suppressed by the $Z$ vector coupling proportional to $1-4 \sin ^{2} \theta_{W}$. The contribution of the diagrams in fig. 4 to the EDM $d_{f}$ of a fermion with electric charge $Q_{f}$ and mass $m_{f}$ is

$$
\begin{gathered}
\frac{d_{f}}{e}=\frac{\alpha Q_{f} m_{f} g K_{\mathrm{QED}}}{32 \sqrt{2} \pi^{3} M_{W} m_{H}^{2}} \operatorname{Im} \sum_{i=1}^{2}\left(\tilde{g}_{d}^{*} U_{i 2} V_{i 1}+\tilde{g}_{u}^{*} U_{i 1} V_{i 2}\right) m_{\chi_{i}^{+}} f\left(\frac{m_{H}^{2}}{m_{\chi_{i}^{+}}^{2}}\right) \\
f(x)=\frac{2 \sqrt{x}}{\sqrt{x-4}}\left[\ln x \ln \frac{\sqrt{x-4}+\sqrt{x}}{\sqrt{x-4}-\sqrt{x}}+\operatorname{Li}_{2}\left(\frac{2 \sqrt{x}}{\sqrt{x}-\sqrt{x-4}}\right)-\operatorname{Li}_{2}\left(\frac{2 \sqrt{x}}{\sqrt{x}+\sqrt{x-4}}\right)\right]
\end{gathered}
$$

${ }^{3}$ If the heavy Higgs fields integrated out at the scale $\tilde{m}$ violate $\mathrm{CP}$, the couplings $\tilde{g}_{u}^{(\prime)}$ and $\tilde{g}_{d}^{(\prime)}$ can in principle have independent phases. In this case, there is a third CP-violating invariant, given by $\operatorname{Im}\left(\tilde{g}_{u} \tilde{g}_{u}^{\prime *} \tilde{g}_{d}^{*} \tilde{g}_{d}^{\prime}\right)$. However, this invariant vanishes, if the $\mathrm{CP}$ violation appears only in the parameter $\beta$ defined in eq. (121) since, in this case, $\arg \left(\tilde{g}_{u}\right)=\arg \left(\tilde{g}_{u}^{\prime}\right)$ and $\arg \left(\tilde{g}_{d}\right)=\arg \left(\tilde{g}_{d}^{\prime}\right)$. 

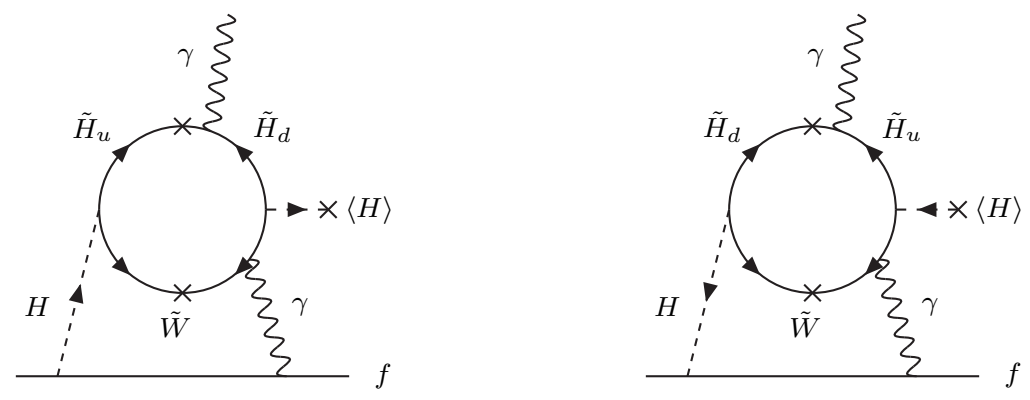

Figure 4: Feynman diagrams contributing to the fermion EDM in Split Supersymmetry. To better illustrate the structure of the interactions, we consider current eigenstates with insertions of $M_{2}, \mu$, and $\langle H\rangle$ denoted by crosses. Two other diagrams with reversed directions of chargino arrows are not shown.

$$
=(2-\ln x) x+\left(\frac{5}{3}-\ln x\right) \frac{x^{2}}{6}+\mathcal{O}\left(x^{3}\right) .
$$

Here $K_{\mathrm{QED}}$ is the leading-logarithm QED correction in the running from the scale of the heavy particles to $m_{f}$ (or $m_{n}$ for the neutron EDM) 39

$$
K_{\mathrm{QED}}=1-\frac{4 \alpha}{\pi} \ln \frac{m_{H}}{m_{f}} .
$$

We work in a general basis in which $\tilde{g}_{u}, \tilde{g}_{d}, M_{2}$, and $\mu$ are all complex. The matrices $U$ and $V$ are defined such that $U^{*} \mathcal{M}_{\chi^{+}} V^{\dagger}$ is diagonal with real and positive entries, where $\mathcal{M}_{\chi^{+}}$is the chargino mass matrix

$$
\mathcal{M}_{\chi^{+}}=\left(\begin{array}{cc}
M_{2} & \sqrt{2} M_{W} \tilde{g}_{u} / g \\
\sqrt{2} M_{W} \tilde{g}_{d} / g & \mu
\end{array}\right) .
$$

We can explicitly write the matrices $U$ and $V$ as

$$
\begin{gathered}
U=\left(\begin{array}{cc}
c_{R} e^{i \phi_{1}} & s_{R} e^{i\left(\phi_{1}-\delta_{R}\right)} \\
-s_{R} e^{i \phi_{2}} & c_{R} e^{i\left(\phi_{2}-\delta_{R}\right)}
\end{array}\right) \quad V=\left(\begin{array}{cc}
c_{L} & s_{L} e^{-i \delta_{L}} \\
-s_{L} & c_{L} e^{-i \delta_{L}}
\end{array}\right) \\
\tan 2 \theta_{L, R}=\frac{2\left|X_{L, R}\right|}{1+\left|X_{R, L}\right|^{2}-\left|X_{L, R}\right|^{2}}, \quad e^{i \delta_{L, R}}=\frac{X_{L, R}}{\left|X_{L, R}\right|}, \\
X_{L}=\frac{\sqrt{2} M_{W}\left(\tilde{g}_{u}^{*} M_{2}+\tilde{g}_{d} \mu^{*}\right)}{g\left(\left|M_{2}\right|^{2}-|\mu|^{2}\right)}, \quad X_{R}=\frac{\sqrt{2} M_{W}\left(\tilde{g}_{d}^{*} M_{2}+\tilde{g}_{u} \mu^{*}\right)}{g\left(\left|M_{2}\right|^{2}-|\mu|^{2}\right)},
\end{gathered}
$$

where $s_{L, R} \equiv \sin \theta_{L, R}$ and $c_{L, R} \equiv \cos \theta_{L, R}$. The phases $\phi_{1}$ and $\phi_{2}$ are chosen such that $m_{\chi_{i}^{+}}$ are real and positive. Using the diagonalization properties of the matrices $U$ and $V$, we 
obtain our final expression for the fermion EDM:

$$
\frac{d_{f}}{e}=\frac{\alpha Q_{f} m_{f} g K_{\mathrm{QED}}}{32 \sqrt{2} \pi^{3} M_{W} m_{H}^{2}} \operatorname{Im}\left(\tilde{g}_{d}^{*} c_{L} s_{R} e^{-i \delta_{R}}+\tilde{g}_{u}^{*} c_{R} s_{L} e^{-i \delta_{L}}\right) e^{i \phi_{1}} m_{\chi_{1}^{+}}\left[f\left(\frac{m_{H}^{2}}{m_{\chi_{1}^{+}}^{2}}\right)-f\left(\frac{m_{H}^{2}}{m_{\chi_{2}^{+}}^{2}}\right)\right] .
$$

Notice that $d_{f}$ vanishes if $m_{\chi_{1}^{+}}=m_{\chi_{2}^{+}}$. Indeed, if the two mass eigenvalues are equal, than $\left|X_{L}\right|=\left|X_{R}\right|=0$, and this implies that the CP invariant $\operatorname{Im}\left(\tilde{g}_{u}^{*} \tilde{g}_{d}^{*} M_{2} \mu\right)$ vanishes.

To have a better understanding of this result, we expand eq. (129) in small $X_{L, R}$. This expansion is valid for chargino masses larger than $M_{W}$, and not degenerate $\left(\left|M_{2}-\mu\right|>M_{W}\right)$. In this case, we have $s_{L, R} \simeq\left|X_{L, R}\right|, \exp \left(i \phi_{1}\right) \simeq M_{2} /\left|M_{2}\right|, m_{\chi_{1}^{+}}=\left|M_{2}\right|$ and $m_{\chi_{2}^{+}}=|\mu|$. Thus

$$
\frac{d_{f}}{e}=\frac{\alpha Q_{f} m_{f} K_{\mathrm{QED}} \operatorname{Im}\left(\tilde{g}_{u}^{*} \tilde{g}_{d}^{*} M_{2} \mu\right)}{16 \pi^{3} m_{H}^{2}\left(\left|M_{2}\right|^{2}-|\mu|^{2}\right)}\left[f\left(\frac{m_{H}^{2}}{\left|M_{2}\right|^{2}}\right)-f\left(\frac{m_{H}^{2}}{|\mu|^{2}}\right)\right]+\mathcal{O}\left(\frac{M_{W}^{2}}{\left|M_{2} \mu\right|}\right)
$$

Notice that eq. (130) is exactly proportional to the CP-violating invariant. We can further simplify the result by considering the limit $\left|M_{2}\right|,|\mu|>m_{H}$ :

$$
\frac{d_{f}}{e}=-\frac{\alpha Q_{f} m_{f} K_{\mathrm{QED}} \tilde{g}_{u} \tilde{g}_{d} \sin \Phi}{16 \pi^{3} M_{2} \mu}\left(\ln \frac{M_{2} \mu}{m_{H}^{2}}+2+\frac{M_{2}^{2}+\mu^{2}}{M_{2}^{2}-\mu^{2}} \ln \frac{\mu}{M_{2}}\right)+\mathcal{O}\left(\frac{M_{W}^{2}}{M_{2} \mu}, \frac{m_{H}^{2}}{M_{2} \mu}\right) .
$$

Here (and in the following) all variables indicate the absolute value of the corresponding quantity and $\Phi=\arg \left(\tilde{g}_{u}^{*} \tilde{g}_{d}^{*} M_{2} \mu\right)$.

The result in eq. (131) can be rederived with a simple argument. By making a chiral rotation of the chargino fields we can eliminate all phases in the mass matrix in eq. (125). Since this transformation is anomalous, it generates a term in the Lagrangian

$$
\frac{e^{2}}{16 \pi^{2}} \arg \left(\operatorname{det} \mathcal{M}_{\chi^{+}}\right) F_{\mu \nu} \tilde{F}^{\mu \nu}
$$

We can expand $\arg \left(\operatorname{det} \mathcal{M}_{\chi^{+}}\right)$in powers of the Higgs background field using $M_{W} \rightarrow g|H| / \sqrt{2}$ in eq. (125),

$$
\arg \left[\operatorname{det} \mathcal{M}_{\chi^{+}}(H)\right]=\arg \left(M_{2} \mu\right)+\frac{\tilde{g}_{u} \tilde{g}_{d} \sin \Phi}{M_{2} \mu} H^{\dagger} H+\mathcal{O}\left(H^{4}\right) .
$$

Therefore, the chiral rotation has induced the operator

$$
\begin{gathered}
\mathcal{O}_{H}=\frac{c_{H}}{\Lambda^{2}} H^{\dagger} H F_{\mu \nu} \tilde{F}^{\mu \nu}, \\
c_{H}=\frac{\alpha}{4 \pi} \tilde{g}_{u} \tilde{g}_{d} \sin \Phi, \quad \Lambda^{2}=M_{2} \mu .
\end{gathered}
$$

Through QED renormalization, the operator $\mathcal{O}_{H}$ mixes with the EDM operator [40, generating

$$
\frac{d_{f}}{e}=-\frac{Q_{f} m_{f} c_{H}}{4 \pi^{2} \Lambda^{2}} \ln \frac{\Lambda^{2}}{m_{H}^{2}}
$$




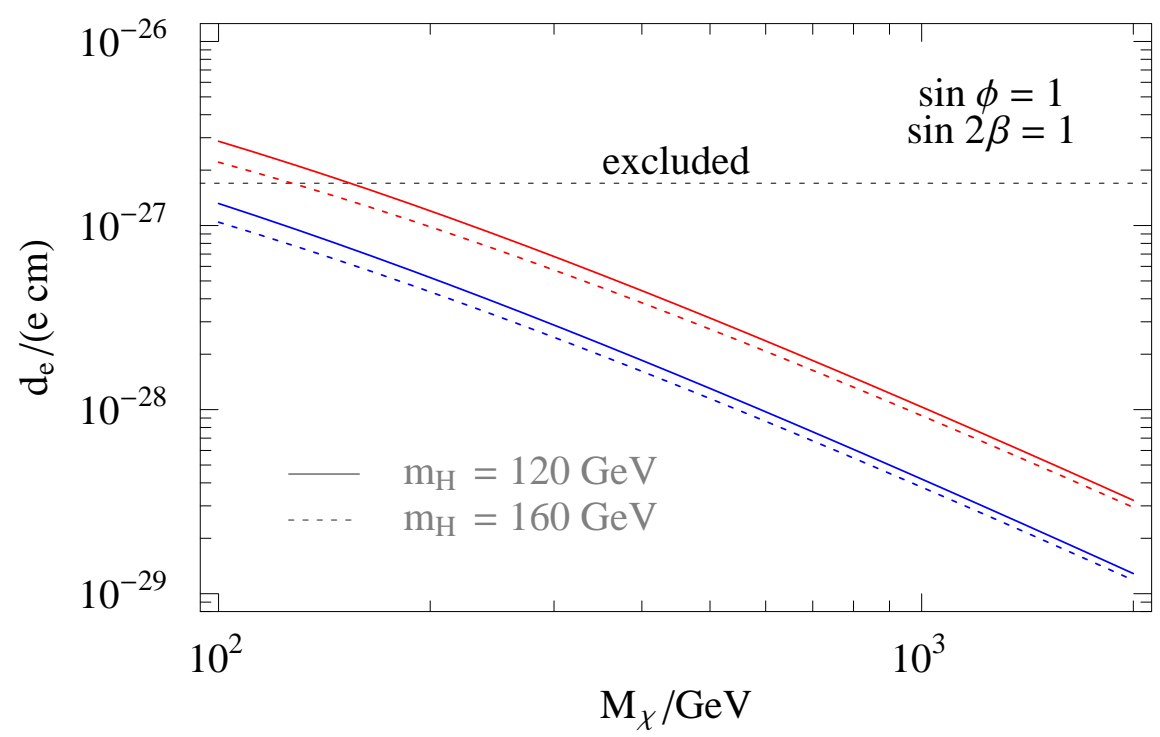

Figure 5: The prediction of the electron EDM in Split Supersymmetry. We plot $d_{e} /(\sin \Phi \sin 2 \beta)$ as a function of the lightest chargino mass $m_{\chi_{1}^{+}}$. The CP-violating phase is $\Phi=\arg \left(\tilde{g}_{u}^{*} \tilde{g}_{d}^{*} M_{2} \mu\right)$ and we have used eq. (121) at the chargino mass scale. Solid lines correspond to $m_{H}=120 \mathrm{GeV}$ and dashed lines to $m_{H}=160 \mathrm{GeV}$. The top two lines correspond to $m_{\chi_{2}^{+}} / m_{\chi_{1}^{+}}=1.5$ and the bottom two lines to $m_{\chi_{2}^{+}} / m_{\chi_{1}^{+}}=4$. The horizontal line shows the present limit $d_{e}<1.7 \times 10^{-27} e \mathrm{~cm}$ at $95 \%$ CL [11].

This correctly reproduces the leading-logarithm behaviour of eq. (131).

The prediction for the electron EDM in Split Supersymmetry is shown in fig. 5 taking the relations in eq. (121) to be approximately valid at the chargino mass scale. The deviation from a straight-line behaviour of the curves in fig. 5 is a result of the logarithmic enhancement explained above. For weak-scale chargino masses and a maximal CP-violating phase, the result is very close to the present experimental limit $d_{e}<1.7 \times 10^{-27} e \mathrm{~cm}$ at 95\% CL [41]. In ordinary low-energy supersymmetry, EDMs are generated at one loop and therefore small phases $\left(\lesssim 10^{-2}\right.$ ) are necessary to reconcile theory with experiments. Because of the two-loop suppression, Split Supersymmetry makes the exciting prediction that EDMs are on the verge of being experimentally tested, if phases take their most natural value of order unity.

EDM experiments are therefore at the frontier of testing Split Supersymmetry. They may reveal hints of new physics even before the start of the LHC. Ongoing and next generation experiments plan to improve the EDM sensitivity by several orders of magnitude within a few years. For example, DeMille and his Yale group [42] will use the molecule PbO to improve the sensitivity of the electron EDM to $10^{-29} e \mathrm{~cm}$ within three years, and possibly to $10^{-31} \mathrm{e} \mathrm{cm}$ within five years. Lamoreaux and his Los Alamos group [43] developed a 
solid state technique that can improve the sensitivity to the electron EDM by $10^{4}$ to reach $10^{-31} \mathrm{e} \mathrm{cm}$. By operating at a lower temperature it is feasible to eventually reach a sensitivity of $10^{-35} \mathrm{e} \mathrm{cm}$, an improvement of eight orders of magnitude over the present sensitivity. The time scale for these is uncertain, as it is tied to funding prospects. Semertzidis and his Brookhaven group [44 plan to trap muons in storage rings and increase the sensitivity of their EDM measurement by five orders of magnitude. A new measurement has been presented by the Sussex group [45]. A number of other experiments aim for an improvement in sensitivity by one or two orders of magnitude, and involve nuclear EDMs.

Improving the EDM measurements by two or more orders of magnitude will test Split Supersymmetry as well as traditional supersymmetric unified theories with low-energy supersymmetry. In the latter, even if all the soft supersymmetry breaking terms are real and consequently do not give rise to CP-violation, the normal Kobayashi-Maskawa phase can be transmitted from the quark to the lepton sector because of quark-lepton unification. In addition, the large flavor breaking, mostly due to the large top mass, results in non-universal slepton and squark mass matrices [46]. As a result, these theories predict electron and quark EDMs near the present limits. Improvement of the sensitivity by two or more orders of magnitude will be a significant test of these theories.

In Split Supersymmetry, CP violation in SM-fermion processes occurs only at two loops. However, since charginos and neutralinos have tree-level couplings with Higgs and gauge bosons, we expect one-loop $\mathrm{CP}$ violation in processes involving $H, W, Z$ and $\gamma$. One such process is the CP-violating Higgs decay into two photons, generated by the operator in eq. (134). The rate is

$$
\begin{gathered}
\frac{\Gamma(H \rightarrow \gamma \gamma)_{\mathrm{CP}^{-}}}{\Gamma(H \rightarrow \gamma \gamma)_{\mathrm{CP}^{+}}}=\left(\frac{\operatorname{Im} I}{\operatorname{Re} I}\right)^{2} \\
I=\frac{4}{3} F_{1 / 2}\left(\frac{4 m_{t}^{2}}{m_{H}^{2}}\right)+F_{1}\left(\frac{4 M_{W}^{2}}{m_{H}^{2}}\right)+\frac{\sqrt{2}}{g} \sum_{i=1}^{2}\left(\tilde{g}_{d}^{*} U_{i 2} V_{i 1}+\tilde{g}_{u}^{*} U_{i 1} V_{i 2}\right) \frac{M_{W}}{m_{\chi_{i}^{+}}} F_{1 / 2}\left(\frac{4 m_{\chi_{i}^{+}}^{2}}{m_{H}^{2}}\right) \\
F_{1 / 2}(x)=-2 x\left[1+(1-x)\left(\arcsin \frac{1}{\sqrt{x}}\right)^{2}\right] \\
F_{1}(x)=2+3 x\left[1+(2-x)\left(\arcsin \frac{1}{\sqrt{x}}\right)^{2}\right]
\end{gathered}
$$

Using the same procedure we followed for the EDM, we can simplify eq. (138) in the limit of heavy, but non-degenerate, charginos. For $m_{H}<2 M_{W}$, we obtain

$$
\operatorname{Im} I=\frac{4 M_{W}^{2} \tilde{g}_{u} \tilde{g}_{d} M_{2} \mu \sin \Phi}{g^{2}\left(M_{2}^{2}-\mu^{2}\right)}\left[M_{2}^{-2} F_{1 / 2}\left(\frac{4 M_{2}^{2}}{m_{H}^{2}}\right)-\mu^{-2} F_{1 / 2}\left(\frac{4 \mu^{2}}{m_{H}^{2}}\right)\right]+\mathcal{O}\left(\frac{M_{W}^{2}}{M_{2} \mu}\right)
$$




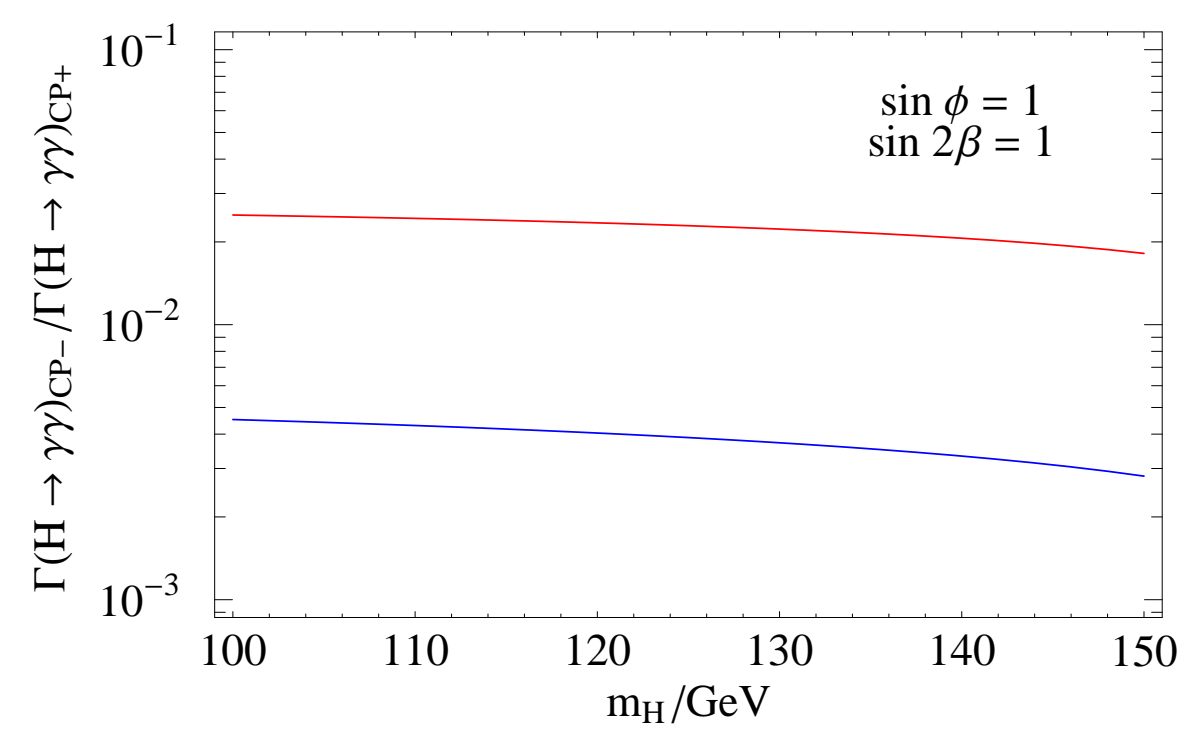

Figure 6: $\Gamma(H \rightarrow \gamma \gamma)_{\mathrm{CP}^{-}} / \Gamma(H \rightarrow \gamma \gamma)_{\mathrm{CP}^{+}}$as a function of the Higgs mass. We take $\sin \Phi=1$ with $\Phi=\arg \left(\tilde{g}_{u}^{*} \tilde{g}_{d}^{*} M_{2} \mu\right)$ and $\sin 2 \beta=1$ using eq. (121) at the chargino mass scale. The top line corresponds to $m_{\chi_{1}^{+}}=100 \mathrm{GeV}$ and $m_{\chi_{2}^{+}}=200 \mathrm{GeV}$, while the bottom line corresponds to $m_{\chi_{1}^{+}}=150 \mathrm{GeV}$ and $m_{\chi_{2}^{+}}=300 \mathrm{GeV}$.

$$
=\frac{16 M_{W}^{2} \tilde{g}_{u} \tilde{g}_{d} \sin \Phi}{3 g^{2} M_{2} \mu}+\mathcal{O}\left(\frac{M_{W}^{2}}{M_{2} \mu}, \frac{m_{H}^{2}}{M_{2} \mu}\right) .
$$

In fig. [6 we show the ratio $\Gamma(H \rightarrow \gamma \gamma)_{\mathrm{CP}^{-}} / \Gamma(H \rightarrow \gamma \gamma)_{\mathrm{CP}^{+}}$, which takes interesting values for light charginos. The scaling with chargino masses of the result shown in fig. [6 can be easily read from eq. (141).

Measurements of the polarization of the photons from Higgs decay is experimentally challenging. However, the $\mathrm{CP}$ quantum number of the Higgs boson can be measured at a $\gamma \gamma$ collider [47. The Higgs production amplitude in the photon-fusion CP-even channel is proportional $\vec{\epsilon}_{1} \cdot \vec{\epsilon}_{2}$, where $\vec{\epsilon}_{1,2}$ are the polarization vectors of the two photon beams. On the other hand, the amplitude in the CP-odd channel is proportional to $\vec{\epsilon}_{1} \times \vec{\epsilon}_{2} \cdot \vec{k}_{\gamma}$, where $\vec{k}_{\gamma}$ is the photon momentum. By varying the linear polarization of the colliding photon beams, one can test the existence of the CP-odd Higgs-photon couplings induced by the chargino loop. In Split Supersymmetry the prospects are particularly favorable, since phases of order one are presently allowed. Also notice that in Split Supersymmetry we have a one-to-one relation between the prediction of the EDM and of the CP-violating Higgs decay.

If charginos and neutralinos are produced at colliders, experiments can directly test the presence of CP-violating phases. One effect of these phases is to modify the relations between model parameters and observables, even for CP-conserving processes. Comparison between 
masses, cross sections and distributions can single out the need to introduce phases for the underlying parameters. However, it will be crucial to test the phases in CP-violating processes. At a linear collider, one can study asymmetries in the CP-odd spin-momentum product $\vec{s}_{\chi_{i}} \cdot \vec{k}_{e^{-}} \times \vec{k}_{\chi_{i}}$ in $e^{+} e^{-} \rightarrow \chi_{i} \chi_{j}$ (for a recent study see [48] and references therein). In Split Supersymmetry, we can take advantage of the order-unity phases allowed by EDM, but we lack the tree-level interference with amplitudes induced by slepton exchange.

\section{Proton Decay}

In this section we discuss proton decay in Split Supersymmetry with SU(5) unification. The contribution from the model-dependent dimension-5 operator is suppressed by four powers of the supersymmetry-breaking scale $\tilde{m}$ and is subdominant for $\tilde{m} \gtrsim 100 \mathrm{TeV}$, if (as is commonly assumed) the amplitude is suppressed by two light quark/lepton masses. In Split Supersymmetry the dimension-5 p-decay operator does not need to be suppressed by light fermion masses, since the heavy squarks can provide an adequate suppression. This can allow for new theoretical possibilities. For example, Yukawa couplings (flavor) can originate in the right-handed fermion sector and there will be no mass suppressions associated with the dimension-5 operator $q q q \ell$, constructed out of only left-handed fermions. In such theories the dimension-5 p-decay would depend on the masses of left-handed squarks and gaugino/higgsinos, and would be highly model-dependent. We now focus on the dimension-6 operator contribution from heavy gauge-boson exchange, which is model independent.

The dominant decay mode is $p \rightarrow e^{+} \pi^{0}$ and the decay width is [49]

$$
\tau_{p}^{-1} \equiv \Gamma\left(p \rightarrow e^{+} \pi^{0}\right)=\frac{\pi m_{p}}{4 f_{\pi}^{2}} \alpha_{N}^{2}(1+D+F)^{2}\left(\frac{\alpha_{\mathrm{GUT}}}{M_{V}^{2}}\right)^{2}\left[A_{R}^{2}+A_{L}^{2}\left(1+\left|V_{u d}\right|^{2}\right)^{2}\right]
$$

where $M_{V}$ is the GUT gauge-boson mass and $\alpha_{\mathrm{GUT}}$ is the unification coupling. Also, $f_{\pi}=$ $131 \mathrm{MeV}$ is the pion decay constant, $(1+D+F)=2.25$ is the chiral Lagrangian factor and we use the lattice result $\alpha_{N}=0.015 \mathrm{GeV}^{3}$ for the hadronic matrix element parameter $\alpha_{N}$ [50]. Note that the lattice determination is 3-5 times larger than the smallest estimate among QCD model calculations. The factors $A_{L}, A_{R}$ account for the leading-log renormalization of the operators $\mathcal{O}_{L}=\left(u_{c}^{\dagger} \sigma_{\mu} q_{1}\right)\left(e_{c}^{\dagger} \sigma^{\mu} q_{1}\right), \mathcal{O}_{R}=\left(u_{c}^{\dagger} \sigma_{\mu} q_{1}\right)\left(d_{c}^{\dagger} \sigma^{\mu} \ell_{1}\right)$ (in Weyl notation). They can be decomposed into four parts,

$$
A_{L, R}=A_{\mathrm{QCD}} A_{\mathrm{SM}}^{L, R} A_{\mathrm{SSSM}}^{L, R} A_{\mathrm{MSSM}}^{L, R}
$$

The factor $A_{\mathrm{QCD}}$ accounts for the QCD renormalization-group evolution below $M_{Z}$. Since 
the matrix elements are evaluated at the scale $Q=2.3 \mathrm{GeV}$, we have

$$
A_{\mathrm{QCD}}=\left[\frac{\alpha_{3}\left(m_{b}\right)}{\alpha_{3}\left(M_{Z}\right)}\right]^{\frac{6}{23}}\left[\frac{\alpha_{3}(Q)}{\alpha_{3}\left(m_{b}\right)}\right]^{\frac{6}{25}} \simeq 1.25 .
$$

The SM running up to the $R$-breaking scale (gaugino and higgsino masses) and the subsequent running up to the supersymmetry breaking scale $\tilde{m}$ give [51]

$$
\begin{aligned}
A_{\mathrm{SM}, \mathrm{SSSM}}^{L} & =\left[\frac{\alpha_{3}(\tilde{m})}{\alpha_{3}\left(M_{Z}\right)}\right]^{\frac{2}{b_{3}}}\left[\frac{\alpha_{2}(\tilde{m})}{\alpha_{2}\left(M_{Z}\right)}\right]^{\frac{9}{4 b_{2}}}\left[\frac{\alpha_{1}(\tilde{m})}{\alpha_{1}\left(M_{Z}\right)}\right]^{\frac{23}{20 b_{1}}} \\
A_{\mathrm{SM}, \mathrm{SSSM}}^{R} & =\left[\frac{\alpha_{3}(\tilde{m})}{\alpha_{3}\left(M_{Z}\right)}\right]^{\frac{2}{b_{3}}}\left[\frac{\alpha_{2}(\tilde{m})}{\alpha_{2}\left(M_{Z}\right)}\right]^{\frac{9}{4 b_{2}}}\left[\frac{\alpha_{1}(\tilde{m})}{\alpha_{1}\left(M_{Z}\right)}\right]^{\frac{11}{20 b_{1}}} .
\end{aligned}
$$

Here $b_{i}$ are the $\beta$-function coefficients $b_{i}=-8 \pi^{2} d g_{i}^{-} 2 / d \ln \mu$ and $\left(b_{1}, b_{2}, b_{3}\right)_{\mathrm{SM}}=(41 / 10,-19 / 6,-7)$ and $\left(b_{1}, b_{2}, b_{3}\right)_{\mathrm{SSSM}}=(9 / 2,-7 / 6,-5)$. Above the supersymmetry breaking scale $\tilde{m}$, the 4 fermion operators mix with their supersymmetric analogs involving scalars, giving [52] (the $\mathrm{U}(1)$ coefficients have been calculated in ref. [53])

$$
\begin{aligned}
& A_{\mathrm{MSSM}}^{L}=\left[\frac{\alpha_{3}\left(M_{\mathrm{GUT}}\right)}{\alpha_{3}(\tilde{m})}\right]^{\frac{4}{3 b_{3}}}\left[\frac{\alpha_{2}\left(M_{\mathrm{GUT}}\right)}{\alpha_{2}(\tilde{m})}\right]^{\frac{3}{2 b_{3}}}\left[\frac{\alpha_{1}\left(M_{\mathrm{GUT}}\right)}{\alpha_{1}(\tilde{m})}\right]^{\frac{23}{30 b_{1}}} \\
& A_{\mathrm{MSSM}}^{R}=\left[\frac{\alpha_{3}\left(M_{\mathrm{GUT}}\right)}{\alpha_{3}(\tilde{m})}\right]^{\frac{4}{3 b_{3}}}\left[\frac{\alpha_{2}\left(M_{\mathrm{GUT}}\right)}{\alpha_{2}(\tilde{m})}\right]^{\frac{3}{2 b_{3}}}\left[\frac{\alpha_{1}\left(M_{\mathrm{GUT}}\right)}{\alpha_{1}(\tilde{m})}\right]^{\frac{11}{30 b_{1}}},
\end{aligned}
$$

with $\left(b_{1}, b_{2}, b_{3}\right)_{\text {MSSM }}=(33 / 5,1,-3)$. The expressions above can be further factorized in order to take into account the thresholds at the EW, $R$, supersymmetry and $\mathrm{SU}(5)$ breaking scales. The appropriate $\beta$-function coefficients can be found in Table 1 .

The proton decay rate crucially depends on the GUT gauge-boson mass $M_{V}$ and on the unified gauge coupling $\alpha_{\mathrm{GUT}}$ at that scale. The two-loop determination of those quantities can be found in ref. [8]. The corresponding prediction for the proton lifetime is shown in fig. [7. lowest panel, as a contour plot in the $\tilde{m}-M_{2}$ plane. We have used $\mu=M_{2}$ and we have obtained $M_{3}$ from $M_{2}\left(M_{\mathrm{GUT}}\right)=M_{3}\left(M_{\mathrm{GUT}}\right)$. The $1 \sigma$ and $2 \sigma$ constraints from $\alpha_{3}\left(M_{Z}\right)=0.119 \pm 0.003$ are also shown. Possible GUT thresholds have been neglected.

The prediction in fig. [7 can be compared with the present $90 \%$ CL limit from SK, $\tau(p \rightarrow$ $\left.e^{+} \pi^{0}\right)>5.0 \times 10^{33} \mathrm{yr}$ [54, with the expected sensitivity after 10 SK live-years, $\tau(p \rightarrow$ $\left.e^{+} \pi^{0}\right) \gtrsim 10^{34} \mathrm{yr}$, and with the expected sensitivity at the next generation of water Cherenkov experiments [55, 56, 57]. For example, a 650 kton UNO-like experiment could reach a sensitivity of $1.6 \times 10^{35} \mathrm{yr}$ in 10 years of running [58].

The dependence of the $p$-decay rate on $\tilde{m}$ is quite mild. Actually, at one loop, $M_{\mathrm{GUT}}$ depends on $\tilde{m}$ only through the heavy Higgs contribution to the $\beta$-functions. This has 

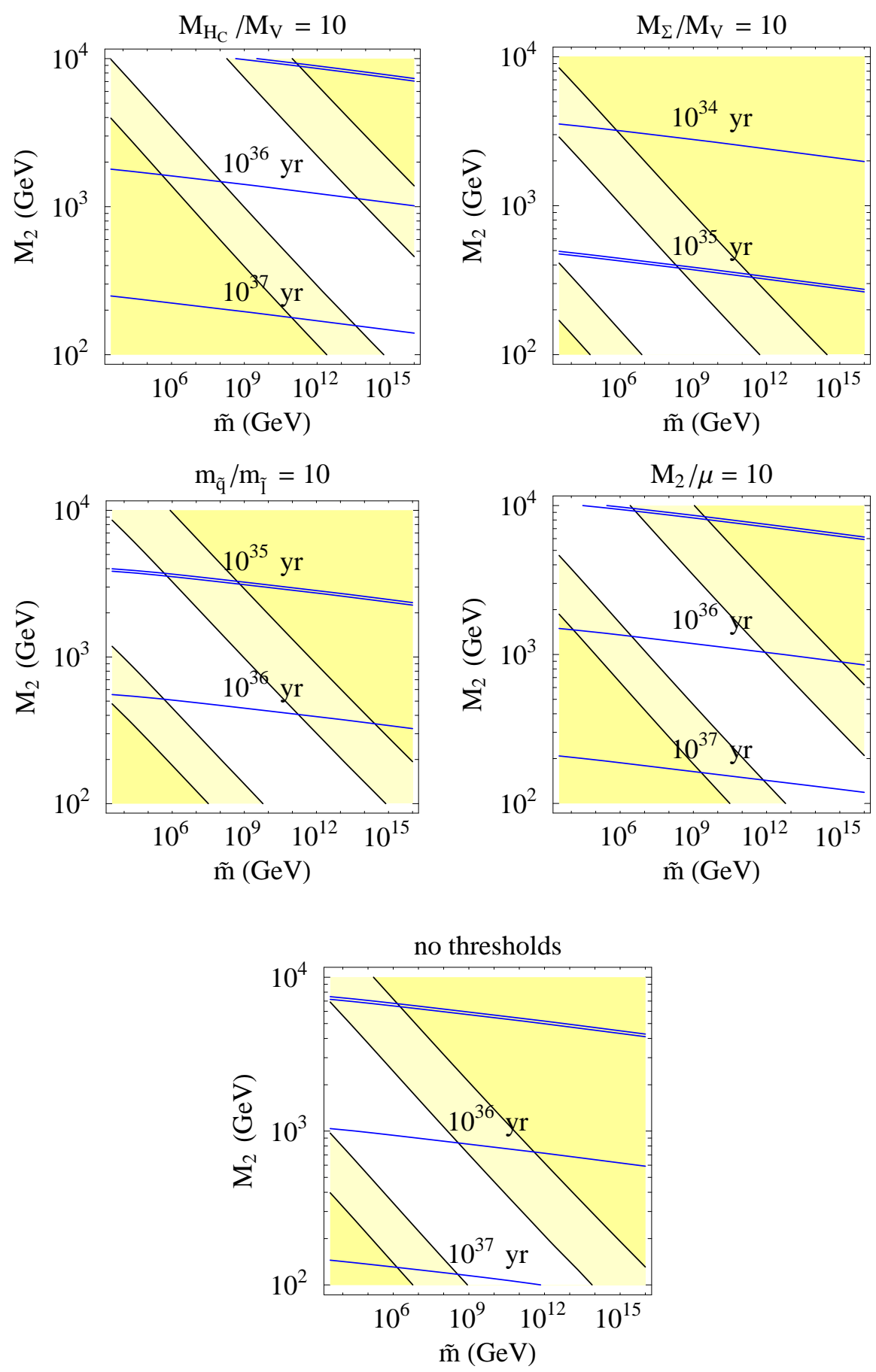

Figure 7: Contours of the proton-decay lifetime, with the thicker line corresponding to $\tau_{p}=10^{35} \mathrm{yr}$. The diagonal bands represent the $1 \sigma$ and $2 \sigma$ constraints corresponding to $\alpha_{3}\left(M_{Z}\right)=0.119 \pm 0.003$. The 4 different thresholds that appear in eqs. (150)-(150) are included as indicated. 
the effect of decreasing $M_{\mathrm{GUT}}$ [8] and therefore of enhancing the $p$-decay rate. However, the effect of $M_{\mathrm{GUT}}$ is partially compensated by the smaller values of $\alpha_{\mathrm{GUT}}$ found in Split Supersymmetry [8]. Notice that additional matter in complete $\mathrm{SU}(5)$ representations at intermediate scales has no effect on $M_{\mathrm{GUT}}$, but increases $\alpha_{\mathrm{GUT}}$. Therefore, a shorter proton lifetime will be found, for instance, in models with intermediate messenger particles, as in gauge mediation or in models with direct mediation, as discussed in sect. 4. We will comment on this possibility at the end of this section.

The proton lifetime becomes shorter as the value of $M_{2}$ increases. Therefore proton-decay searches can be viewed as complementary to direct searches at accelerators, although they have a rather modest reach in parameter space. This figure shows that a correct value of $\alpha_{3}$ implies an upper limit on $M_{2}$ and a lower limit on $\tau_{p}$. As a consequence, larger values of $M_{2}$ and shorter proton lifetimes are allowed for lower values of $\tilde{m}$.

We now investigate the effect of three types of thresholds corrections.

- $R$-breaking scale thresholds due to $\mu \neq M_{2}$.

- supersymmetry-breaking scale thresholds associated to a non-degeneracy among the sfermion masses or between the sfermion masses and the heavy Higgs mass $m_{H}$ (the latter will turn out not to play a role at 1 loop). For simplicity, we will assume that all squarks and all sleptons are separately degenerate with common masses $m_{\tilde{q}}, m_{\tilde{l}}$ respectively.

- SU(5)-breaking scale thresholds. We will consider the effect of the heavy SU(5) vector superfields with mass $M_{V}$, of the color triplet partners of the Higgs doublets with mass $M_{H_{C}}$, and of an adjoint $\mathrm{SU}(5)$-breaking chiral superfield with mass $M_{\Sigma}$.

The one-loop thresholds can be incorporated in the 2-loop evolution of the gauge couplings by using the following matching conditions at the scales $M_{V}, m_{H}$ and $M_{2}$ :

$$
\begin{aligned}
\frac{1}{\alpha_{i}\left(M_{V}-\right)} & =\frac{1}{\alpha_{i}\left(M_{V}+\right)}+\frac{b_{i}^{H_{C}}}{2 \pi} \ln \frac{M_{V}}{M_{H_{C}}}+\frac{b_{i}^{\Sigma}}{2 \pi} \ln \frac{M_{V}}{M_{\Sigma}} \\
\frac{1}{\alpha_{i}\left(m_{H}-\right)} & =\frac{1}{\alpha_{i}\left(m_{H}+\right)}+\frac{b_{i}^{\tilde{q}}}{2 \pi} \ln \frac{m_{\tilde{l}}}{m_{\tilde{q}}}+\frac{b_{i}^{\tilde{f}}}{2 \pi} \ln \frac{m_{H}}{m_{\tilde{l}}} \\
\frac{1}{\alpha_{i}\left(M_{2}-\right)} & =\frac{1}{\alpha_{i}\left(M_{2}+\right)}+\frac{b_{i}^{\tilde{H}}}{2 \pi} \ln \frac{M_{2}}{\mu} .
\end{aligned}
$$

Here, $b_{i}^{x}$ represents the contribution of the particle $x$ to the $\beta$-function coefficient of the gauge coupling $g_{i}$. The numerical values are listed in Table 1. The coefficients $b_{\tilde{f}}=(2,2,2)$ are associated to the whole set of sfermions, in a full $\mathrm{SU}(5)$ representation. Therefore, the $\ln \left(m_{H} / m_{\tilde{l}}\right)$ term does not enter the determination of $M_{V}$ and $\alpha_{3}$ at one loop.

An analytic understanding of the threshold effects on the determination of $M_{\mathrm{GUT}}$ and $\alpha_{3}$ 


\begin{tabular}{|c||c|c|c||c|c|c||c|c|c|}
\hline$x$ & $\tilde{H}$ & $\tilde{W}$ & $\tilde{G}$ & $H$ & $\tilde{q}$ & $\tilde{l}$ & $V$ & $H_{C}$ & $\Sigma$ \\
\hline$b_{1}^{x}$ & $2 / 5$ & 0 & 0 & $1 / 10$ & $11 / 10$ & $9 / 10$ & -10 & $2 / 5$ & 0 \\
\hline$b_{2}^{x}$ & $2 / 3$ & $4 / 3$ & 0 & $1 / 6$ & $3 / 2$ & $1 / 2$ & -6 & 0 & 2 \\
\hline$b_{3}^{x}$ & 0 & 0 & 2 & 0 & 2 & 0 & -4 & 1 & 3 \\
\hline
\end{tabular}

Table 1: Contributions to the $\beta$-function coefficients from particle $x$. The $\beta$-function coefficients for a theory with the particle content of SM $+\{x\}$ (where $\{x\}$ describes the set of new particles) are obtained by the relation $b_{i}=b_{i}^{\mathrm{SM}}+\sum_{x} b_{i}^{x}$, where $b_{i}^{\mathrm{SM}}=(41 / 10,-19 / 6,-7)$ and $b_{i}^{x}$ are given in the table.

can be easily gained in the one loop approximation,

$$
\begin{aligned}
\delta \ln M_{V} & =-\frac{1}{14} \ln \frac{M_{V}}{M_{H_{C}}}+\frac{5}{14} \ln \frac{M_{V}}{M_{\Sigma}}+\frac{1}{14} \ln \frac{m_{\tilde{l}}}{m_{\tilde{q}}}+\frac{1}{21} \ln \frac{M_{2}}{\mu} \\
\delta \alpha_{3}^{-1} & =\frac{1}{2 \pi}\left(\frac{9}{7} \ln \frac{M_{V}}{M_{H_{C}}}-\frac{3}{7} \ln \frac{M_{V}}{M_{\Sigma}}+\frac{3}{14} \ln \frac{m_{\tilde{l}}}{m_{\tilde{q}}}-\frac{6}{7} \ln \frac{M_{2}}{\mu}\right), \\
\delta \alpha_{\mathrm{GUT}}^{-1} & =-\frac{1}{2 \pi}\left(-\frac{1}{14} \log \frac{M_{V}}{M_{H_{C}}}+\frac{33}{14} \log \frac{M_{V}}{M_{\Sigma}}+\frac{11}{7} \log \frac{m_{\tilde{l}}}{m_{\tilde{q}}}+\frac{5}{7} \log \frac{M_{2}}{\mu}+2 \log \frac{m_{H}}{m_{\tilde{l}}}\right)
\end{aligned}
$$

where $\delta x$ denotes the contribution to $x$ due to the thresholds. A positive contribution $\delta \alpha_{3}^{-1}$ lowers the $\alpha_{3}$ bands in fig. [7, while a positive contribution $\delta \ln M_{V}$ rises the lifetime contours. This result is evident in the figures, which however contains the full 2-loop effects.

From the first panel of fig. [7 we observe that the Higgs triplet has a significant effect on $\alpha_{3}$, and a limited effect on $\tau_{p}$. For heavy $H_{C}$, it is possible to obtain $\tau_{p}<10^{35} \mathrm{yr}$, but only at the price of very heavy gauginos. The second panel of fig. 7 shows that $\Sigma$ has a considerable effect on $\tau_{p}$, but not on $\alpha_{3}$. For a heavy $\Sigma$, proton decay is well within the reach of a megaton water Cherenkov experiment, even for gaugino masses accessible to the LHC and CLIC. The third panel shows that having squarks heavier than sleptons improves the chances for proton-decay searches. We should remark however that squarks much heavier than sleptons is not consistent with the simplest unification relations. The fourth panel illustrates how a threshold with higgsinos lighter than gauginos modifies the $\alpha_{3}$ prediction, allowing for larger proton-decay rates. The latter plot illustrates a particularly interesting and fairly generic case where the $p$-decay rate may be observable and consistent with both unification and DM abundance, for a broad range of scalar masses. It corresponds to $M_{2} \sim 10 \mathrm{TeV}$ and $\mu \sim 1 \mathrm{TeV}$. This is the situation we have if $\mu$ starts out much smaller than the gaugino masses at the UV scale. Then, the physical gaugino masses will be roughly an order of magnitude heavier than the low-energy value of $\mu$ [ ], leading to a mostly-higgsino LSP - which must then weigh about a $\mathrm{TeV}$ to be the DM, leading to the parameter choice of the fourth plot of fig. [7. This suggests that potentially observable $p$-decay will be a fairly common characteristic of theories which start out with a small $\mu$ in the UV. On the other 
hand, it will be hard to see such a $\sim \mathrm{TeV}$-mass Higgsino at the LHC.

Finally, we consider the effect of additional matter in complete vectorial SU(5) multiplets. Such extra multiplets arise in simple implementations of direct mediation, see sect. 固 The leading effect, which we calculate, is due to the increase in $\alpha_{\mathrm{GUT}}$. Note that the extra multiplets also affect the operator renormalization coefficients $A_{L, R}$ at one loop and the determination of $M_{\mathrm{GUT}}$ at two loops. The effect on $\alpha_{\mathrm{GUT}}$ is given by

$$
\frac{1}{\alpha_{\mathrm{GUT}}}=\left(\frac{1}{\alpha_{\mathrm{GUT}}}\right)_{0}-\frac{N}{2 \pi} \log \frac{M_{\mathrm{GUT}}}{\tilde{m}},
$$

where the index "0" refers to the determination of $\alpha_{\mathrm{GUT}}$ in the absence of extra matter, which corresponds to the predictions shown in Fig. 7 and $N$ is the Dynkin index of the gauge representation of the extra matter $(N=1$ for a single $5+\overline{5}, N=3$ for a single $10+\overline{10})$. In this simple approximation, the perturbativity limit on $N$ is about 15 for $\tilde{m}=10^{11} \mathrm{GeV}$ and scales with $1 / \log \left(M_{\mathrm{GUT}} / \tilde{m}\right)$. The limit on $N$ from proton decay is approximately the same. If the Dynkin index $N$ is sizeable but not fine-tuned to be close to the perturbativity limit, we can expect an increase of $\alpha_{\mathrm{GUT}}$ by a factor $\mathcal{O}(1)$, which leads to an increase of the proton decay rate by a factor $\mathcal{O}(1)^{2}$.

In conclusion, some versions of Split Supersymmetry, such as those with higgsino LSP, naturally allow for the possibility of potentially observable proton lifetime, consistent with unification and DM.

\section{Conclusions}

In this paper we have investigated theoretical, cosmological and phenomenological aspects of Split Supersymmetry.

We have shown that the mass spectrum of Split Supersymmetry, which was originally motivated by low-energy considerations (dark matter and prediction of $\alpha_{s}$ ), can actually have a natural justification in the high-energy theory. An approximate $R$-symmetry, which forbids gaugino and higgsino mass terms, can explain the hierarchies of the mass spectrum of Split Supersymmetry. Nevertheless, Split Supersymmetry can emerge from the high-energy theory not necessarily as a consequence of an imposed $R$-symmetry, but simply as a result of the pattern of supersymmetry breaking. Using the property that $R$-symmetric soft terms correspond to dimension- 2 operators and $R$-breaking soft terms to dimension-3 operators, we have shown that supersymmetry $D$-breaking leads to Split Supersymmetry, while $F$ breaking leads to the usual mass spectrum with no hierarchies among sparticles. In the case 
of $D$-breaking, the underlying $R$-symmetry protecting higgsino and gaugino masses emerges as an accidental symmetry, much alike the approximate lepton-number conservation that protects neutrino masses in the Standard Model.

We have also studied the general structure of Split Supersymmetry as we vary the mechanism of supersymmetry breaking or, ultimately, the gravitino mass $m_{3 / 2}$. Since the squark and slepton mass scale $\tilde{m}$ is an arbitrary parameter, no longer tied to the weak scale, the theory offers novel scenarios with different theoretical, cosmological and phenomenological implications. In general, the different features of Split Supersymmetry are best parametrized by the values of $m_{3 / 2}$ and $\tilde{m}$.

Whenever $m_{3 / 2}$ is much larger than the weak scale (which, in particular, is the case for gravity mediation with $\tilde{m} \gg \mathrm{TeV}$ ), then anomaly-mediated contribution can upset the hierarchy between supersymmetric scalars and fermions. This issue has been addressed in sect. 3. We have found that in theories where supersymmetry breaking is intimately tied to gravity, such that supersymmetry is restored in the $M_{\mathrm{Pl}} \rightarrow \infty$ limit, the anomaly-mediated contributions are suppressed and there is no obstacle to considering $m_{3 / 2}$ far above the TeV scale.

We have also discussed an interesting option, available in Split Supersymmetry but not in low-energy supersymmetry, to break supersymmetry and directly communicate it to squarks and sleptons at tree-level, via renormalizable couplings. These theories naturally generate a large hierarchy between the scalars and the gauginos/Higgsinos, and also permit an interesting spectrum with a gravitino LSP. Such models inevitably require extra charged matter at intermediate scales, raising the value of the unified gauge coupling at the GUT scale, and possible enhancing the proton decay rate from dimension 6 operators to observable levels.

In Split Supersymmetry, the gravitino mass can vary in a much larger range than in ordinary low-energy supersymmetry and this has new cosmological implications. In sect. 5] we have revisited gravitino production in the early universe. The decay of thermalized heavy particles with mass $\tilde{m}$ plays a new and important role, creating a gravitino abundance which is independent of the reheat temperature $T_{R}$, and which depends only on physical particle masses.

Heavy gravitinos eventually decay into supersymmetric particles. A peculiarity of gravitinos with masses much larger than the weak scale (and thus a peculiarity of Split Supersymmetry) is that the decay occurs well before the beginning of nucleosynthesis, and therefore the gravitino abundance before decay is not tightly constrained. If the decay happens after neutralinos have decoupled from the thermal bath, then a non-thermal population of neu- 
tralinos is generated. We have studied the range of parameters for which this non-thermal $\chi$ component can account for the observed dark-matter.

The existence of a non-thermal neutralino population has important phenomenological consequences. The dark-matter argument is a critical ingredient of Split Supersymmetry, providing a tight constraint on the higgsino and gaugino parameters. The non-thermal $\chi$ density gives us more freedom, allowing us to consider neutralino parameters that are normally excluded, because they lead to an insufficient thermal density. In spite of this freedom, the upper bound on $m_{\chi}$, derived from thermal dark-matter considerations, is not evaded, but actually reinforced. This is essential, as the bound on $m_{\chi}$ provides the necessary link between gaugino-higgsino masses and the weak scale.

If the scale of supersymmetry mediation is much smaller than $M_{\mathrm{Pl}}$, the gravitino can be light, and it can even become the LSP. In this case, the neutralino can no longer form the dark matter, and the connection between its mass and the weak scale disappears (although an upper bound on its mass still exists, under the condition that gravitinos constitute the dark matter). A gravitino LSP is a viable dark-matter candidate. The collider signals of this case are also quite distinctive, whenever the decays into gravitinos occur inside the detector. A peculiar signature of Split Supersymmetry with light gravitinos is the gluino decay $\tilde{g} \rightarrow \tilde{G} g$. This decay can dominate over the ordinary 3-body process into neutralinos and charginos, when the scale of supersymmetry-breaking mediation is close to $\tilde{m}$. This is the case of direct mediation.

Next, we have addressed some phenomenological issues. Split Supersymmetry solves the flavour and CP problem, by considering very heavy squarks and sleptons. However, the effective theory below the scale $\tilde{m}$ contains a $\mathrm{CP}$ violating phase. This leads to predictions for electron and neutron EDMs, which are just beyond the present experimental limits, for a phase of order unity and for weak-scale chargino masses. The difference with respect to ordinary low-energy supersymmetry, where phases have to be smaller than about $10^{-2}$ to be consistent with present limits, is that EDM are only generated at two loops in Split Supersymmetry. Because of the intense experimental activity aiming at improving the EDM measurements, our prediction offers the exciting possibility that Split Supersymmetry could start revealing itself even before the LHC is operational. Other tests of CP violation at colliders are also possible, as discussed in sect. 6]

Finally, we have investigated how Split Supersymmetry affects proton decay. Dimension-5 operators are naturally suppressed, because of the heavy squarks. Since Split Supersymmetry has the effect of reducing the value of the unification mass, proton-decay dimension- 6 
operators can be enhanced. However, in the minimal version of the model, the unification coupling constant increases, nearly compensating the effect of a lower $M_{G U T}$. Low-energy thresholds can enhance the proton-decay rate, and this is especially true if we consider very heavy higgsinos and gauginos. In this sense, searches for proton decay can be complementary to collider searches, since they are most sensitive to a region of sparticle masses hard to test at colliders.

In conclusion, we have shown that Split Supersymmetry can naturally emerge from a fundamental high-energy theory. It has specific implications for the origin of the dark matter and it makes the exciting prediction that EDMs are naturally within the reach of ongoing experiments.

We acknowledge communications and discussions with D. DeMille, H. Haber, L. Ibañez, S. Lamoreaux, M. Luty, R. Rattazzi, A. Ritz, Y. Semertzidis and E. Witten.

\section{References}

[1] R. Bousso and J. Polchinski, JHEP 0006 (2000) 006 arXiv:hep-th/0004134; A. Maloney, E. Silverstein and A. Strominger, arXiv:hep-th/0205316 S. Kachru, R. Kallosh, A. Linde and S. P. Trivedi, Phys. Rev. D 68, 046005 (2003) arXiv:hep-th/0301240; L. Susskind, arXiv:hep-th/0302219; M. R. Douglas, JHEP 0305, 046 (2003) arXiv:hep-th/0303194|; T. Banks, M. Dine and E. Gorbatov, JHEP 0408 (2004) 058 arXiv:hep-th/0309170; S. B. Giddings, S. Kachru and J. Polchinski, Phys. Rev. D 66, 106006 (2002) arXiv:hep-th/0105097; S. Ashok and M. R. Douglas, JHEP 0401, 060 (2004) arXiv:hep-th/0307049; F. Denef and M. R. Douglas, JHEP 0405, 072 (2004) arXiv:hep-th/0404116 ; A. Giryavets, S. Kachru and P. K. Tripathy, JHEP 0408, 002 (2004) arXiv:hep-th/0404243; J. P. Conlon and F. Quevedo, arXiv:hep-th/0409215 O. DeWolfe, A. Giryavets, S. Kachru and W. Taylor, to appear.

[2] S. Weinberg, Phys. Rev. Lett. 59, 2607 (1987). For earlier related work see T. Banks, Nucl. Phys. B 249, 332 (1985) and A. D. Linde, in "300 Years of Gravitation" (Editors: S.Hawking and W. Israel, Cambridge University Press, 1987), 604. This constraint was sharpened in A. Vilenkin, Phys. Rev. Lett. 74, 846 (1995) arXiv:gr-qc/9406010. A nice review of these ideas can be found in C. J. Hogan, Rev. Mod. Phys. 72, 1149 (2000) arXiv:astro-ph/9909295 and M. J. Rees, arXiv:astro-ph/0401424. 
[3] M. R. Douglas, arXiv:hep-ph/0401004 L. Susskind, arXiv:hep-th/0405189 M. R. Douglas, arXiv:hep-th/0405279 M. Dine, E. Gorbatov and S. Thomas, arXiv:hep-th/0407043 E. Silverstein, arXiv:hep-th/0407202, M. R. Douglas, arXiv:hep-th/0409207.

[4] S. Dimopoulos and H. Georgi, Nucl. Phys. B 193 (1981) 150.

[5] S. Dimopoulos, S. Raby and F. Wilczek, Phys. Rev. D 24 (1981) 1681.

[6] H. Goldberg, Phys. Rev. Lett. 50 (1983) 1419.

[7] N. Arkani-Hamed and S. Dimopoulos, arXiv:hep-th/0405159.

[8] G. F. Giudice and A. Romanino, arXiv:hep-ph/0406088

[9] A. Arvanitaki, C. Davis, P. W. Graham and J. G. Wacker, arXiv:hep-ph/0406034, A. Pierce, arXiv:hep-ph/0406144 S. h. Zhu, arXiv:hep-ph/0407072 B. Mukhopadhyaya and S. SenGupta, arXiv:hep-th/0407225; W. Kilian, T. Plehn, P. Richardson and E. Schmidt, arXiv:hep-ph/0408088; R. Mahbubani, arXiv:hep-ph/0408096 M. Binger, arXiv:hep-ph/0408240 J. L. Hewett, B. Lillie, M. Masip and T. G. Rizzo, arXiv:hep-ph/0408248; L. Anchordoqui, H. Goldberg and C. Nunez, arXiv:hep-ph/0408284 K. Cheung and W. Y. Keung, arXiv:hep-ph/0408335.

[10] A. E. Nelson and N. Seiberg, Nucl. Phys. B 416 (1994) 46 arXiv:hep-ph/9309299.

[11] G. F. Giudice and A. Masiero, Phys. Lett. B 206 (1988) 480.

[12] L. Randall and R. Sundrum, Nucl. Phys. B 557, 79 (1999) arXiv:hep-th/9810155; G. F. Giudice, M. A. Luty, H. Murayama and R. Rattazzi, JHEP 9812, 027 (1998) arXiv:hep-ph/9810442.

[13] M. A. Luty and N. Okada, hep-th/0209178.

[14] D. E. Kaplan and N. Weiner, hep-ph/0108001.

[15] M. A. Luty, Phys. Rev. Lett. 89 (2002) 141801 arXiv:hep-th/0205077.

[16] S. Ferrara, L. Girardello and F. Palumbo, Phys. Rev. D 20 (1979) 403.

[17] T. Moroi, H. Murayama and M. Yamaguchi, Phys. Lett. B 303 (1993) 289.

[18] M. Bolz, A. Brandenburg and W. Buchmuller, Nucl. Phys. B 606 (2001) 518 arXiv:hep-ph/0012052. 
[19] P. Fayet, Phys. Lett. B 86 (1979) 272.

[20] D. N. Spergel et al. [WMAP Collaboration], Astrophys. J. Suppl. 148 (2003) 175 arXiv:astro-ph/0302209.

[21] R. Barbieri, M. Frigeni and G. F. Giudice, Nucl. Phys. B 313 (1989) 725.

[22] J. Gasser, H. Leutwyler and M. E. Sainio, Phys. Lett. B 253 (1991) 252; D. Adams et al. [Spin Muon Collaboration], Phys. Lett. B 357 (1995) 248.

[23] G. F. Giudice, E. W. Kolb and A. Riotto, Phys. Rev. D 64, 023508 (2001) arXiv:hep-ph/0005123.

[24] T. Gherghetta, G. F. Giudice and J. D. Wells, Nucl. Phys. B 559, 27 (1999) arXiv:hep-ph/9904378.

[25] S. Dimopoulos, R. Esmailzadeh, L. J. Hall and G. D. Starkman, Nucl. Phys. B 311 (1989) 699.

[26] M. H. Reno and D. Seckel, Phys. Rev. D 37, 3441 (1988); K. Kohri, Phys. Rev. D 64, 043515 (2001) arXiv:astro-ph/0103411.

[27] M. Kawasaki, K. Kohri and T. Moroi, arXiv:astro-ph/0402490.

[28] M. Y. Khlopov and A. D. Linde, Phys. Lett. B 138, 265 (1984); J. R. Ellis, J. E. Kim and D. V. Nanopoulos, Phys. Lett. B 145, 181 (1984); J. R. Ellis, G. B. Gelmini, J. L. Lopez, D. V. Nanopoulos and S. Sarkar, Nucl. Phys. B 373, 399 (1992).

[29] R. H. Cyburt, J. R. Ellis, B. D. Fields and K. A. Olive, Phys. Rev. D 67, 103521 (2003) arXiv:astro-ph/0211258.

[30] B. D. Fields and K. A. Olive, Astrophys. J. 506, 177 (1998) arXiv:astro-ph/9803297.

[31] Y. I. Izotov and T. X. Thuan, Astrophys. J. 602, 200 (2004) arXiv:astro-ph/0310421.

[32] D. Kirkman, D. Tytler, N. Suzuki, J. M. O’Meara and D. Lubin, Astrophys. J. Suppl. 149, 1 (2003) arXiv:astro-ph/0302006.

[33] G. F. Giudice, I. Tkachev and A. Riotto, JHEP 9908, 009 (1999) arXiv:hep-ph/9907510; JHEP 9911, 036 (1999) arXiv:hep-ph/9911302; R. Kallosh, L. Kofman, A. D. Linde and A. Van Proeyen, Phys. Rev. D 61, 103503 (2000) arXiv:hep-th/9907124; Class. Quant. Grav. 17, 4269 (2000) arXiv:hep-th/0006179. 
[34] M. Kawasaki and T. Moroi, Prog. Theor. Phys. 93 (1995) 879 arXiv:hep-ph/9403364; T. Gherghetta, G. F. Giudice and A. Riotto, Phys. Lett. B 446, 28 (1999) arXiv:hep-ph/9808401; T. Asaka, K. Hamaguchi and K. Suzuki, Phys. Lett. B 490, 136 (2000) arXiv:hep-ph/0005136.

[35] J. R. Ellis, K. A. Olive, Y. Santoso and V. C. Spanos, Phys. Lett. B 588, 7 (2004) arXiv:hep-ph/0312262; J. L. Feng, S. Su and F. Takayama, arXiv:hep-ph/0404231 L. Roszkowski and R. R. de Austri, arXiv:hep-ph/0408227.

[36] M. Dine and A. E. Nelson, Phys. Rev. D 48, 1277 (1993) arXiv:hep-ph/9303230; M. Dine, A. E. Nelson and Y. Shirman, Phys. Rev. D 51, 1362 (1995) arXiv:hep-ph/9408384; G. F. Giudice and R. Rattazzi, Phys. Rept. 322, 419 (1999) arXiv:hep-ph/9801271.

[37] S. Weinberg, Phys. Rev. Lett. 63, 2333 (1989); E. Braaten, C. S. Li and T. C. Yuan, Phys. Rev. Lett. 64, 1709 (1990).

[38] S. M. Barr and A. Zee, Phys. Rev. Lett. 65, 21 (1990) [Erratum-ibid. 65, 2920 (1990)]; D. Chang, W. Y. Keung and A. Pilaftsis, Phys. Rev. Lett. 82, 900 (1999) [Erratum-ibid. 83, 3972 (1999)] arXiv:hep-ph/9811202; D. Chang, W. F. Chang and W. Y. Keung, Phys. Rev. D 66, 116008 (2002) arXiv:hep-ph/0205084; A. Pilaftsis, Nucl. Phys. B 644, 263 (2002) arXiv:hep-ph/0207277.

[39] G. Degrassi and G. F. Giudice, Phys. Rev. D 58, 053007 (1998) arXiv:hep-ph/9803384.

[40] X. Zhang and B. L. Young, Phys. Rev. D 49 (1994) 563 arXiv:hep-ph/9309269; A. Lue, K. Rajagopal and M. Trodden, Phys. Rev. D 56, 1250 (1997) arXiv:hep-ph/9612282.

[41] B. C. Regan, E. D. Commins, C. J. Schmidt and D. DeMille, Phys. Rev. Lett. 88 (2002) 071805 .

[42] D. Kawall, F. Bay, S. Bickman, Y. Jiang and D. DeMille, electron in AIP Conf. Proc. 698 (2004) 192.

[43] S. K. Lamoreaux, other arXiv:nucl-ex/0109014.

[44] Y. K. Semertzidis, arXiv:hep-ex/0401016.

[45] J. J. Hudson, B. E. Sauer, M. R. Tarbutt and E. A. Hinds, Phys. Rev. Lett. 89 (2002) 023003 arXiv:hep-ex/0202014. 
[46] S. Dimopoulos and L. J. Hall, Phys. Lett. B 344 (1995) 185 arXiv:hep-ph/9411273; R. Barbieri, L. J. Hall and A. Strumia, Nucl. Phys. B 449 (1995) 437 arXiv:hep-ph/9504373.

[47] B. Grzadkowski and J. F. Gunion, Phys. Lett. B 294 (1992) 361 arXiv:hep-ph/9206262; M. Kramer, J. H. Kuhn, M. L. Stong and P. M. Zerwas, Z. Phys. C 64 (1994) 21 arXiv:hep-ph/9404280.

[48] S. Y. Choi, M. Drees and B. Gaissmaier, Phys. Rev. D 70 (2004) 014010 arXiv:hep-ph/0403054.

[49] P. Langacker, Phys. Rept. 72 (1981) 185.

[50] S. Aoki et al. [JLQCD Collaboration], Phys. Rev. D 62, 014506 (2000) arXiv:hep-lat/9911026.

[51] A. J. Buras, J. R. Ellis, M. K. Gaillard and D. V. Nanopoulos, Nucl. Phys. B 135 (1978) 66. J. R. Ellis, M. K. Gaillard and D. V. Nanopoulos, Phys. Lett. B 88 (1979) 320. F. Wilczek and A. Zee, Phys. Rev. Lett. 43 (1979) 1571.

[52] L. E. Ibanez and C. Munoz, Nucl. Phys. B 245 (1984) 425.

[53] C. Munoz, Phys. Lett. B 177 (1986) 55.

[54] Y. Suzuki et al. [TITAND Working Group Collaboration], arXiv:hep-ex/0110005.

[55] Y. Itow et al., arXiv:hep-ex/0106019.

[56] C. K. Jung, arXiv:hep-ex/0005046.

[57] M. V. Diwan et al., arXiv:hep-ex/0306053.

[58] A. Rubbia, arXiv:hep-ph/0407297.

[59] J. Hisano, H. Murayama and T. Yanagida, Nucl. Phys. B 402, 46 (1993) arXiv:hep-ph/9207279; J. Hisano, arXiv:hep-ph/0004266. 